\title{
Design, synthesis, and biological activity of novel 1,2,4-oxadiazole derivatives
}

\author{
Lingzhi Zhu, Huanan Zeng, Dan Liu, Yun Fu, Qiong Wu, Baoan Song* and Xiuhai Gan* (1)
}

\begin{abstract}
Background: Plant diseases seriously threaten food security, it is urgent to discover efficient and low-risk chemical pesticides. 1,2,4-Oxadiazole derivatives exhibit broad spectrum of agricultural biological activities. For discovering novel molecules with excellent agricultural activities, novel 1,2,4-oxadiazole derivatives were synthesized and evaluated for their agricultural activities.

Result: Bioassays results showed that the title compounds exhibited moderate nematocidal activity against Meloidogyne incognita and anti-fungal activity to Rhizoctonia solani. It's worth noting that compounds $\mathbf{5 m}, \mathbf{5 r}, \mathbf{5 u}, \mathbf{5 v}, \mathbf{5 x}$ and $\mathbf{5 y}$ showed strong antibacterial effects on Xanthomonas oryzae pv. oryzae (Xoo), with $\mathrm{EC}_{50}$ values of 36.25, 24.14, $28.82,19.44,25.37$ and $28.52 \mu \mathrm{g} / \mathrm{mL}$, respectively, superior to bismerthiazol (BMT, EC ${ }_{50}=77.46 \mu \mathrm{g} / \mathrm{mL}$ ) and thiodiazole copper (TDC, $\mathrm{EC}_{50}=99.31 \mathrm{\mu g} / \mathrm{mL}$ ). Compounds $\mathbf{5 p}$, $\mathbf{5 u}$ and $\mathbf{5} \mathbf{v}$ exhibited excellent antibacterial ability against Xanthomonas oryzae pv. oryzicola (Xoc), with $\mathrm{EC}_{50}$ values of $31.40,19.04$ and $21.78 \mu \mathrm{g} / \mathrm{mL}$, respectively, better than that of BMT $\left(E C_{50}=68.50 \mu \mathrm{g} / \mathrm{mL}\right)$ and TDC $\left(E_{50}=91.05 \mu \mathrm{g} / \mathrm{mL}\right)$. In addition, compound $\mathbf{5 v}$ exerted moderate antibacterial effects on rice bacterial leaf blight.

Conclusions: Twenty-six novel 1,2,4-oxadiazole derivatives were obtained and their biological activities were evaluated. Compound $\mathbf{5 u}$ and $\mathbf{5 v}$ exhibited excellent antibacterial activity $X_{O O}$ and $X_{O} c$. These results indicated that 1,2,4-oxadiazole derivatives containing a trifluoromethyl pyridine moiety could be as potential alternative templates for discovering novel antibacterial agents.
\end{abstract}

Keywords: Synthesis, 1,2,4-Oxadiazole derivatives, Trifluoromethyl pyridine, Antibacterial activity, Nematocidal activity

\section{Introduction}

Crop plants are constantly challenged by a wide variety of pathogens which threaten their growth and survival, such as bacteria, fungus, and plant-parasitic nematodes. As two rice bacterial diseases, rice bacterial leaf blight and rice bacterial leaf streaks caused by Xanthomonas oryzae pv. oryzae (Xoo) and Xanthomonas oryzae pv. oryzicola $(X o c)$, respectively, serious impact on every stage of plant

\footnotetext{
*Correspondence: songbaoan22@yahoo.com; gxh200719@163.com State Key Laboratory Breeding Base of Green Pesticide and Agricultural Bioengineering, Key Laboratory of Green Pesticide and Agricultural Bioengineering, Ministry of Education, Guizhou University, Guiyang 550025, China
}

growth and development. These diseases may result in a loss of up to $80 \%$ of the crop and cause severe economic damage [1-4]. Meanwhile, fungal diseases, for example, rice sheath wilt caused by Rhizoctonia solani, still pose a huge threat to global agriculture [5]. In addition, over 3000 plant species are affected by nematodes worldwide, including ornamental flowers, fruit trees, cereals and vegetables [6-8]. The diseases caused by nematodes infecting plants are a serious threat to crop security, causing over $\$ 157$ billion in economic losses to farmers worldwide $[9,10]$. Root-knot nematode is a plant parasitic nematode that affects plant growth by essentially damaging the plant roots $[11,12]$. There is an urgent need to 
devise a method for the effective manual control of these plant diseases, as plants cannot quickly and effectively resist them [13]. Presently, pesticides are often used for agricultural control due to their rapid response to plant diseases [14, 15], however, the long-term abuse of pesticides has led to the emergence of resistance in pathogenic organisms and may pose a risk to human health [16-18]. Therefore, developing novel, highly-efficient, and environmentally benign agents against plant diseases remains a daunting task in pesticide sciences.

Heterocyclic structures are widely used in molecular design, and many commodity medicines have been developed [19], such as tioxazafen (Fig. 1), bismerthiazol and fluopyram. As an important five-membered heterocyclic scaffold, 1,2,4-oxadiazoles, with good potent biological properties $[20,21]$ have been extensively used in pesticide and medicine [22-24] molecule design. Moreover, the 1,2,4-oxadiazole heterocycle is a bioisostere of amide but shows better hydrolytic and metabolic stability [22], it is still used as an important pharmacophore to create novel drug molecules. Meanwhile, 1,3,4-thiadiazol and 1,3,4-oxadiazole have been reported to have good biological activities and were used to design drug molecules in pesticide. In our previous work, we designed and synthesized a series of novel 1,3,4-thiadiazol and 1,3,4-oxadiazole derivatives with effective control of bacterial $[25,26]$, fungal [27-29] and plant-parasitic nematodes [30] diseases. In addition, trifluoromethyl pyridine is an important heterocyclic structure containing fluorine, and also a common group in the current commercial pesticides. Fluopyram, containing a trifluoromethyl pyridine moiety, is not only used for control of fungal diseases, but also is used for control plant-parasitic nematodes disease [31, 32].

From the above standpoints, the compounds containing an 1,2,4-oxadiazole heterocycle, 1,3,4-thiadiazol (1,3,4-oxadiazole), or trifluoromethyl pyridine moiety exhibit broad-spectrum agricultural biological activities, which can be used as pharmacophore to design the novel pesticide. Encouraged by these promising results, and in order to obtain compounds with higher biological activity, we employed the structure-based bioisostere strategy, an excellent tool for lead were introduced into 1,2,4-optimisation, 1,3,4-thiadiazol (1,3,4-oxadiazole) and trifluoromethyl pyridine pharmacophores oxadiazole skeleton to design and synthesize a series of novel 1,2,4-oxadiazole derivatives. Meanwhile their agricultural biological activities, including nematocidal, anti-fungal, and antibacterial activity were roundly evaluated. We aimed to discovery novel structure diversity molecules with broad-spectrum activity for development of new pesticides.

\section{Methods}

\section{Chemistry}

All reagents and chemical materials of the analytically pure were purchased from chemical commercial companies. The reactions were monitored by thin-layer chromatography analysis and the $\mathrm{ZF}_{7}$ ultraviolet analyzer (Yuhua Instrument Co., Ltd. Gong Yi, China). ${ }^{1} \mathrm{H}$ and ${ }^{13} \mathrm{C}$ NMR spectra were obtained on the JEOL-ECX-500 spectrometer (JEOL, Tokyo, Japan) or $400 \mathrm{MHz}$ spectrometer (JEOL, Tokyo, Japan). The melting points of the compounds were measurement by the $\mathrm{X}-4 \mathrm{~B}$ melting point instrument of readings were uncorrected (Yidian Physical Optical Instrument Co., Ltd. Shanghai, China). High-resolution mass spectra (ESI TOF $(+)$ ) were obtained on the LTQ Orbitrap XL (Thermo Scientific, MO, USA).

\section{General synthesis procedure for compounds $5 a-5 i$}

The procedure for synthesizing the target compounds 5a-5i was described in Scheme 1. A mixture of substituted benzonitrile $(5.0 \mathrm{mmol}), \mathrm{NaOH}(3.0 \mathrm{mmol})$ and hydroxylamine hydrochloride $(7.5 \mathrm{mmol})$ in ethanol/water $(30 \mathrm{~mL}, V: V=5: 1)$ was refluxed for $4 \mathrm{~h}$. Then, ethanol was removed and the mixture was extracted with ethyl acetate and removed the solvent to obtain intermediate $\mathbf{1}$. Then chloroacetyl chloride $(2.0 \mathrm{mmol})$ was added to a solution of intermediate $1(2.0 \mathrm{mmol})$ in toluene and resulting mixture stirred for $6-8 \mathrm{~h}$ at $110-120{ }^{\circ} \mathrm{C}$. After complication of the reaction, the solvent was removed and the residue was recrystallized from ethanol to obtain intermediate 2 . Finally, $\mathrm{K}_{2} \mathrm{CO}_{3}(1.0 \mathrm{mmol})$ was added to a solution of corresponding 1,3,4-oxadiazole/thiadiazole thiol intermediate $(1.0 \mathrm{mmol})$ in $\mathrm{MeCN}(20 \mathrm{~mL})$ and stirred at room temperature for $0.5 \mathrm{~h}$. Then intermediate $2(1.0 \mathrm{mmol})$ was added and the mixture was refluxed. After complication

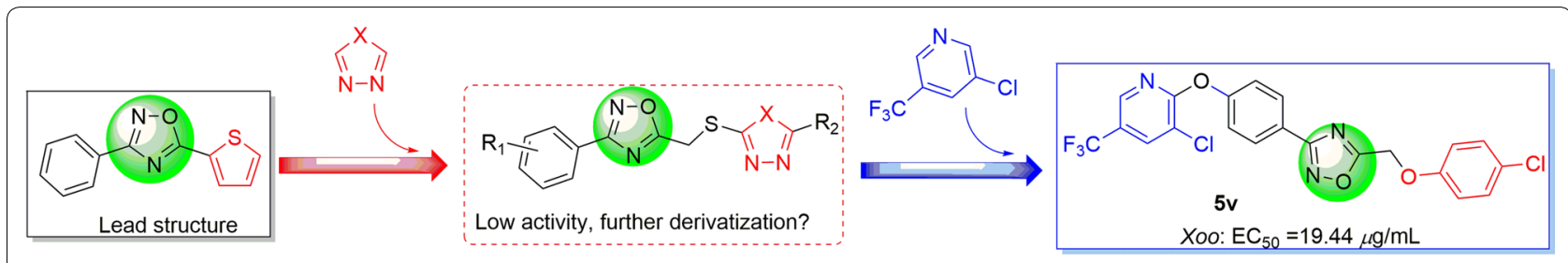

Fig. 1 Design strategy of the target compounds 


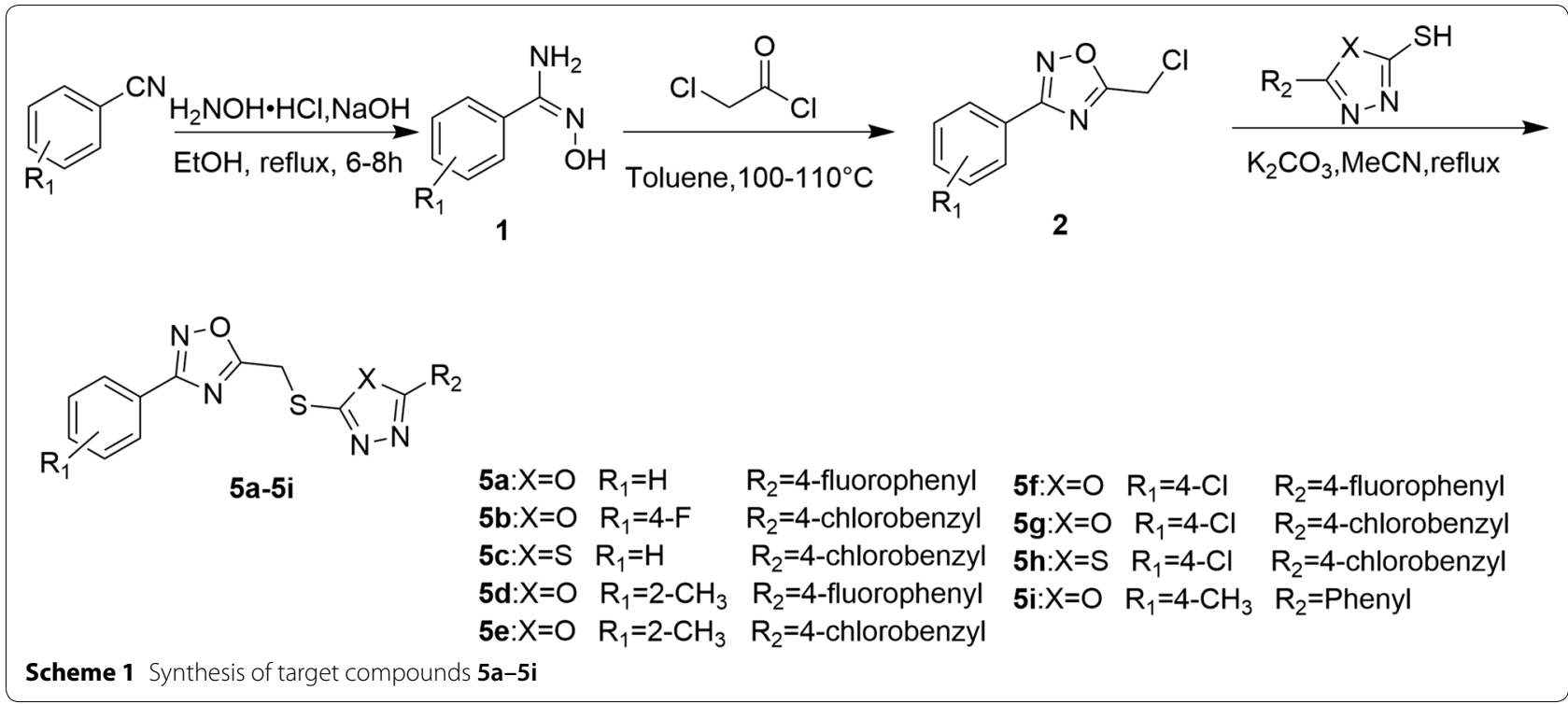

of the reaction, the solvent was removed and the residue was recrystallized from ethanol to obtain the target compounds $\mathbf{5 a}-\mathbf{5 i}$ with $34.8-62.3 \%$ yields.

\section{General synthesis procedure for compounds $5 j-5 r$}

A mixture of 2,3-dichloro-5-(trifluoromethyl) pyridine $(5.0 \mathrm{mmol})$ and 4-hydroxybenzonitrile $(5.0 \mathrm{mmol})$ in DMF $(8 \mathrm{~mL})$ was first stirred at room temperature for $0.5 \mathrm{~h}$. Then $\mathrm{K}_{2} \mathrm{CO}_{3}(10.0 \mathrm{mmol})$ was added and the mixture was refluxed for $8 \mathrm{~h}$. After complication of the reaction, the mixture was poured into $100 \mathrm{~mL}$ of ethanol. The precipitate was filtered off and to obtain intermediate 3 . Then, a mixture of intermediate $3(3.0 \mathrm{mmol})$, $\mathrm{NaHCO}_{3}(3.0 \mathrm{mmol})$ and hydroxylamine hydrochloride $(4.5 \mathrm{mmol})$ in ethanol $(20 \mathrm{~mL})$ was refluxed for $2 \mathrm{~h}$. The solvent was removed and the residue was poured into water. The mixture was extracted with ethyl acetate and removed the solvent to obtain intermediate 4 . Then substituted acyl chloride $(2.0 \mathrm{mmol})$ was added to a solution of intermediate $4(2.0 \mathrm{mmol})$ in toluene and resulting mixture stirred for $6-8 \mathrm{~h}$ at $110-120{ }^{\circ} \mathrm{C}$. After complication of the reaction, the solvent was removed and the residue was recrystallized from ethanol to afford the target compounds $\mathbf{5 j - 5 r}$ with $31.7-62.9 \%$ yields (Scheme 2).

\section{General synthesis procedure for compounds $5 s-5 z$}

$\mathrm{K}_{2} \mathrm{CO}_{3}(1.0 \mathrm{mmol})$ was added to a solution of substituted phenol $(1.0 \mathrm{mmol})$ in $\mathrm{MeCN}(20 \mathrm{~mL})$ and stirred at room temperature for $0.5 \mathrm{~h}$. Then compound $5 \mathrm{r}(1.0 \mathrm{mmol})$ was added and the mixture was refluxed for $4-6 \mathrm{~h}$. After complication of the reaction, the solvent was removed and the residue was recrystallized from ethanol to obtain the target compounds $\mathbf{5 s}-\mathbf{5 z}$ with $41.8-61.8 \%$ yields (Scheme 3).

5-(((5-(4-fluorobenzyl)-1,3,4-oxadiazol-2-yl)thio) methyl)-3-phenyl-1,2,4-oxadiazole (5a) Yellow solid; yield 55.0\%; mp: $106.5-107.1{ }^{\circ} \mathrm{C} ;{ }^{1} \mathrm{H}$ NMR $(400 \mathrm{MHz}$, $\left.\mathrm{CDCl}_{3}\right) \delta 7.47(\mathrm{~d}, J=7.6 \mathrm{~Hz}, 3 \mathrm{H}, \mathrm{Ph}-\mathrm{H}), 7.47(\mathrm{~d}, J=7.6 \mathrm{~Hz}$, $3 \mathrm{H}, \mathrm{Ph}-\mathrm{H}) 7.19$ (d, J=8.6 Hz, 2H, Ph-H), 4.75 (s, 2H, $\left.\mathrm{CH}_{2}-\right) .{ }^{13} \mathrm{C}$ NMR $\left(101 \mathrm{MHz}, \mathrm{CDCl}_{3}\right) \delta 174.47,168.81$, $165.77,162.59,161.69,131.52,129.33,129.33,128.77$, $128.77,127.49,127.49,126.13,119.59,116.62,116.40$, 31.44; HRMS (ESI) calcd for $\mathrm{C}_{17} \mathrm{H}_{12} \mathrm{~N}_{4} \mathrm{O}_{2} \mathrm{SF}[\mathrm{M}+\mathrm{H}]^{+}$: 355.06534, found 355.06595 .

5-(((5-(4-chlorophenyl)-1,3,4-oxadiazol-2-yl)thio) methyl)-3-(4-fluorophenyl)-1,2,4-oxadiazole (5b) Yellow solid; yield 45.0\%; mp: $101.9-103.2{ }^{\circ} \mathrm{C}$; ${ }^{1} \mathrm{H}$ NMR $\left(400 \mathrm{MHz}, \mathrm{CDCl}_{3}\right) \delta 8.05(\mathrm{dd}, J=8.9,5.4 \mathrm{~Hz}, 2 \mathrm{H}, \mathrm{Ph}-\mathrm{H})$, $7.94(\mathrm{~d}, J=8.7 \mathrm{~Hz}, 2 \mathrm{H}, \mathrm{Ph}-\mathrm{H}), 7.48(\mathrm{~d}, J=8.7 \mathrm{~Hz}, 2 \mathrm{H}$, $\mathrm{Ph}-\mathrm{H}), 7.15(\mathrm{t}, J=8.7 \mathrm{~Hz}, 2 \mathrm{H}, \mathrm{Ph}-\mathrm{H}), 4.75(\mathrm{~s}, 2 \mathrm{H},-$ $\left.\mathrm{CH}_{2}-\right) .{ }^{13} \mathrm{C}$ NMR $\left(101 \mathrm{MHz}, \mathrm{CDCl}_{3}\right) \delta 174.96,169.70$, $168.77,162.58161 .73,131.42,129.57,129.57,129.29$, 129.29, 128.89, 128.89, 127.86, 127.86, 127.54, 126.32, 31.46; HRMS (ESI) calcd for $\mathrm{C}_{17} \mathrm{H}_{11} \mathrm{~N}_{4} \mathrm{O}_{2} \mathrm{SFCl}[\mathrm{M}+\mathrm{H}]^{+}$: 389.02698, found 353.02681.

5-(((5-(4-chlorobenzyl)-1,3,4-oxadiazol-2-yl)thio) methyl)-3-phenyl-1,2,4-oxadiazole (5c) Brown solid; yield 57.3\%; mp: 83.5-84.7 ${ }^{\circ} \mathrm{C}$; ${ }^{1} \mathrm{H}$ NMR (400 MHz, $\left.\mathrm{CDCl}_{3}\right) \delta$ 8.06-8.02 (m, 2H, Ph-H), 7.51-7.45 (m, 3H, Ph-H), 7.32$7.27(\mathrm{~m}, 2 \mathrm{H}, \mathrm{Ph}-\mathrm{H}), 7.22(\mathrm{t}, J=6.8 \mathrm{~Hz}, 2 \mathrm{H}, \mathrm{Ph}-\mathrm{H}), 4.78(\mathrm{~s}$, $\left.2 \mathrm{H},-\mathrm{CH}_{2}-\right), 4.35\left(\mathrm{~s}, 2 \mathrm{H},-\mathrm{CH}_{2}-\right) .{ }^{13} \mathrm{C}$ NMR $(101 \mathrm{MHz}$, $\left.\mathrm{CDCl}_{3}\right) \delta 174.85,170.43,168.70,162.95,134.90,133.67$, $131.43,130.15,130.15,129.25,129.25,128.88,128.88$, 


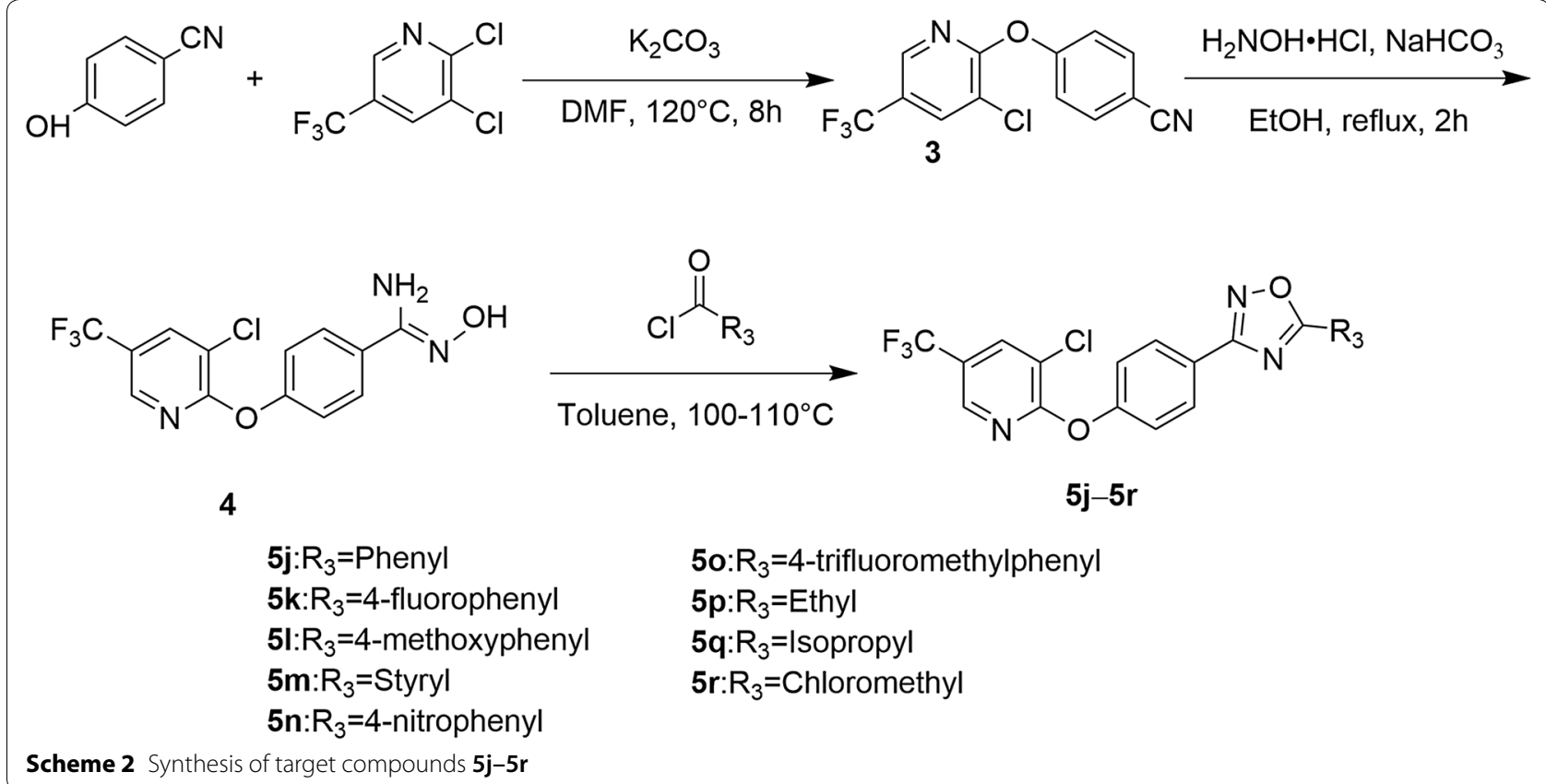

Scheme 2 Synthesis of target compounds $\mathbf{5 j}-\mathbf{5 r}$

Scheme 3 Synthesis of target compounds $\mathbf{5 s} \mathbf{- 5 z}$

127.48, 127.48, 126.27, 35.80, 27.65; HRMS (ESI) calcd for $\mathrm{C}_{18} \mathrm{H}_{14} \mathrm{~N}_{4} \mathrm{OS}_{2} \mathrm{Cl}[\mathrm{M}+\mathrm{H}]^{+}:$401.02856, found 401.02921.

5-(((5-(4-fluorophenyl)-1,3,4-thiadiazol-2-yl)thio) methyl)-3-(o-tolyl)-1,2,4-oxadiazole (5d) Yellow solid; yield 48.6\%; mp: 98.1-98.7 ${ }^{\circ} \mathrm{C}$; ${ }^{1} \mathrm{H}$ NMR $(500 \mathrm{MHz}$, $\left.\mathrm{CDCl}_{3}\right) \delta 7.97-7.92(\mathrm{~m}, 3 \mathrm{H}, \mathrm{Ph}-\mathrm{H}), 7.47(\mathrm{~d}, J=8.7 \mathrm{~Hz}$, 2H, Ph-H), 7.40-7.36 (m, 1H, Ph-H), 7.32-7.26 (m, 2H, Ph-H), 4.76 (s, 2H, $\left.-\mathrm{CH}_{2}-\right), 2.59\left(\mathrm{~s}, 3 \mathrm{H},-\mathrm{CH}_{3}\right) .{ }^{13} \mathrm{C}$ NMR $\left(126 \mathrm{MHz}, \mathrm{CDCl}_{3}\right) \delta 173.38,169.47,165.86,162.06$, 138.42 , 131.54, 130.97, 130.22, 130.22, 129.63, 129.63,
128.16, 126.10, 125.47, 121.83, 114.02, 26.85, 22.28; HRMS (ESI) calcd for $\mathrm{C}_{18} \mathrm{H}_{15} \mathrm{~N}_{4} \mathrm{OS}_{2}[\mathrm{M}+\mathrm{H}]^{+}: 367.06766$, found 367.06818 .

5-(((5-(3-chlorobenzyl)-1,3,4-oxadiazol-2-yl)thio) methyl)-3-(o-tolyl)-1,2,4-oxadiazole (5e) Yellow solid; yield 62.3\%; mp: 82.6-83.2 ${ }^{\circ} \mathrm{C}$; ${ }^{1} \mathrm{H}$ NMR $(500 \mathrm{MHz}$, $\left.\mathrm{CDCl}_{3}\right) \delta 7.94-7.91(\mathrm{~m}, 1 \mathrm{H}, \mathrm{Ph}-\mathrm{H}), 7.39(\mathrm{td}, J=7.6$, 1.4 Hz, 1H, Ph-H), 7.32-7.26 (m, 4H, Ph-H), 7.23-7.19 (m, 2H, Ph-H), 4.67 (s, 2H, $\left.-\mathrm{CH}_{2}-\right), 4.15$ (s, 2H, $-\mathrm{CH}_{2}-$ ), $2.58\left(\mathrm{~s}, 3 \mathrm{H},-\mathrm{CH}_{3}\right) .{ }^{13} \mathrm{C} \mathrm{NMR}\left(126 \mathrm{MHz}, \mathrm{CDCl}_{3}\right) \delta$ 
$173.34,169.40,166.83,162.52,138.43,133.84,131.76$, $131.55,131.55,130.97,130.24,130.24,129.25,129.25$, 126.12, 125.47, 31.35, 26.69, 22.28; HRMS (ESI) calcd for $\mathrm{C}_{19} \mathrm{H}_{16} \mathrm{~N}_{4} \mathrm{O}_{2} \mathrm{SCl}[\mathrm{M}+\mathrm{H}]^{+}:$399.06714, found 399.06770.

3-(4-chlorophenyl)-5-(((5-(4-fluorophenyl)-1,3,4-oxadiazol-2-yl)thio)methyl)-1,2,4-oxadiazole (5f) White solid; yield 60.2\%; mp: 106.6-108.1 ${ }^{\circ} \mathrm{C}$; ${ }^{1} \mathrm{H}$ NMR $(500 \mathrm{MHz}$, $\left.\mathrm{CDCl}_{3}\right) \delta 8.03-7.97(\mathrm{~m}, 4 \mathrm{H}, \mathrm{Ph}-\mathrm{H}), 7.46-7.42(\mathrm{~m}, 2 \mathrm{H}$, Ph-H), 7.21-7.16 (m, 2H, Ph-H), 4.74 (s, $\left.2 \mathrm{H},-\mathrm{CH}_{2}-\right) .{ }^{13} \mathrm{C}$ NMR $\left(126 \mathrm{MHz} \mathrm{CDCl}_{3}\right) \delta 174.75,168.15,165.95,161.70$, $137.79,130.03,130.03,129.59,129.59,129.01,129.01$, 128.91, 128.91, 124.74, 119.65, 116.72, 26.83; HRMS (ESI) calcd for $\mathrm{C}_{17} \mathrm{H}_{11} \mathrm{~N}_{4} \mathrm{O}_{2} \mathrm{SFCl}[\mathrm{M}+\mathrm{H}]+$ : 389.02637, found 389.02698 .

5-(((5-(4-chlorobenzyl)-1,3,4-oxadiazol-2-yl)thio) methyl)-3-(4-chlorophenyl)-1,2,4-oxadiazole (5 g) Yellow solid; yield 56.3\%; mp: 84.7-86.0 ${ }^{\circ} \mathrm{C}$; ${ }^{1} \mathrm{H}$ NMR $\left(500 \mathrm{MHz}, \mathrm{CDCl}_{3}\right) \delta 7.99-7.95(\mathrm{~m}, 2 \mathrm{H}, \mathrm{Ph}-\mathrm{H})$ 7.46-7.43 (m, 2H, Ph-H), 7.32-7.28 (m, 2H, Ph-H), 7.22-7.19 (m, $2 \mathrm{H}, \mathrm{Ph}-\mathrm{H}), 4.77$ (s, 2H, $\left.-\mathrm{CH}_{2}-\right)$, $4.34\left(\mathrm{~s}, 2 \mathrm{H},-\mathrm{CH}_{2}-\right)$. ${ }^{13} \mathrm{C}$ NMR $\left(126 \mathrm{MHz}, \mathrm{CDCl}_{3}\right) \delta 175.22,170.54,168.02$, $162.96,137.69,134.97,133.80,130.26,130.26,129.59$, $129.59,129.14,129.14,128.89,128.89,124.88,35.90$, 27.63; HRMS (ESI) calcd for $\mathrm{C}_{18} \mathrm{H}_{13} \mathrm{~N}_{4} \mathrm{O}_{2} \mathrm{SCl}_{2}[\mathrm{M}+\mathrm{H}]^{+}$: 419.01233, found 419.01308.

5-(((5-(4-chlorobenzyl)-1,3,4-thiadiazol-2-yl)thio) methyl)-3-(4-chlorophenyl)-1,2,4-oxadiazole (5h) Yellow solid; yield 34.8\%; mp: 83.0-84.3 ${ }^{\circ} \mathrm{C}$; ${ }^{1} \mathrm{H}$ NMR $(500 \mathrm{MHz}$, $\left.\mathrm{CDCl}_{3}\right) \delta 7.99-7.93(\mathrm{~m}, 2 \mathrm{H}, \mathrm{Ph}-\mathrm{H}), 7.48-7.40(\mathrm{~m}, 2 \mathrm{H}$, $\mathrm{Ph}-\mathrm{H}), 7.31-7.26(\mathrm{~m}, 2 \mathrm{H}, \mathrm{Ph}-\mathrm{H}), 7.21$ (d, J=8.5 Hz, $2 \mathrm{H}, \mathrm{Ph}-\mathrm{H}), 4.65$ (s, 2H, $\left.-\mathrm{CH}_{2}-\right), 4.15\left(\mathrm{~s}, 2 \mathrm{H},-\mathrm{CH}_{2}-\right)$. ${ }^{13} \mathrm{C}$ NMR $\left(126 \mathrm{MHz}, \mathrm{CDCl}_{3}\right) \delta 174.67,168.08,166.88$, $162.41,137.80,133.87,131.71,130.39,130.39,129.61$, $129.61,129.30,129.30,129.00,128.77,124.71,31.44$, 26.67; HRMS (ESI) calcd for $\mathrm{C}_{18} \mathrm{H}_{13} \mathrm{~N}_{4} \mathrm{OS}_{2} \mathrm{Cl}_{2}[\mathrm{M}+\mathrm{H}]^{+}$: 434.98953, found 434.99023.

5-(((5-(4-chlorophenyl)-1,3,4-thiadiazol-2-yl)thio) methyl)-3-(p-tolyl)-1,2,4-oxadiazole (5i) Yellow solid; yield 41.5\%; mp: $106.7-107.8{ }^{\circ} \mathrm{C}$; ${ }^{1} \mathrm{H}$ NMR $(400 \mathrm{MHz}$, $\left.\mathrm{CDCl}_{3}\right) \delta 7.97-7.92(\mathrm{~m}, 4 \mathrm{H}, \mathrm{Ph}-\mathrm{H}), 7.47(\mathrm{~d}, J=8.6 \mathrm{~Hz}$, 2H, Ph-H), 7.27 (d, J=8.2 Hz, 2H, Ph-H), 4.74 (s, 2H, $\mathrm{CH}_{2}-$ ), $2.41\left(\mathrm{~s}, 3 \mathrm{H},-\mathrm{CH}_{3}\right) .{ }^{13} \mathrm{C}$ NMR (101 MHz, $\mathrm{CDCl}_{3}$ ) $\delta$ 174.16, 165.79, 161.95, 141.94, 138.31, 133.80, 129.52, $129.52,129.59,129.59,128.00,128.00,127.44,127.44$, 123.32 121.75, 26.82, 21.62; HRMS (ESI) calcd for $\mathrm{C}_{18} \mathrm{H}_{15} \mathrm{~N}_{4} \mathrm{OS}_{2}[\mathrm{M}+\mathrm{H}]^{+}:$367.06763, found 367.06818.

3-(4-((3-chloro-5-(trifluoromethyl)pyridin-2-yl)oxy) phenyl)-5-phenyl-1,2,4-oxadiazole (5j) White solid; yield 31.7\%; mp: $94.5-95.0{ }^{\circ} \mathrm{C}$; ${ }^{1} \mathrm{H}$ NMR (400 MHz, DMSO- $d_{6}$ ) $\delta 8.63(\mathrm{~d}, J=2.2 \mathrm{~Hz}, 1 \mathrm{H}$, Pyridine-H), $8.56(\mathrm{dd}, J=2.1$, $0.9 \mathrm{~Hz}, 1 \mathrm{H}$, Pyridine-H), 8.23-8.17 (m, 4H, Ph-H), 7.797.74 (m, 1H, Ph-H), 7.71-7.66 (m, 2H, Ph-H), 7.52-7.48 (m, 2H, Ph-H). ${ }^{13} \mathrm{C}$ NMR (101 MHz, DMSO- $\left.d_{6}\right) \delta 175.99$,
$168.17,160.92,155.64,143.64,137.85,133.92,130.09$, $130.09,129.36,129.36,128.43,124.79,124.06,123.81$, 123.09, 123.09, 122.52, 122.14, 119.16; HRMS (ESI) calcd for $\mathrm{C}_{20} \mathrm{H}_{12} \mathrm{~N}_{3} \mathrm{O}_{2} \mathrm{~F}_{3} \mathrm{Cl}[\mathrm{M}+\mathrm{H}]^{+}$: 418.05539, found 418.05647.

3-(4-((3-chloro-5-(trifluoromethyl)pyridin-2-yl) oxy)phenyl)-5-(4-fluorophenyl)-1,2,4-oxadiazole (5k) White solid; yield 41.8\%; mp: $106.5-107.3{ }^{\circ} \mathrm{C}$; ${ }^{1} \mathrm{H}$ NMR $\left(400 \mathrm{MHz}, \mathrm{DMSO}-d_{6}\right) \delta 8.64(\mathrm{~d}, J=2.1 \mathrm{~Hz}, 1 \mathrm{H}$, Pyridine$\mathrm{H}), 8.56(\mathrm{dd}, J=2.1,0.9 \mathrm{~Hz}, 1 \mathrm{H}$, Pyridine-H), 8.23-8.17 (m, 4H, Ph-H), 7.79-7.74 (m, 1H, Ph-H), 7.78-7.74 (m, 2H, Ph-H), 7.54-7.47 (m, 2H, Ph-H). ${ }^{13} \mathrm{C}$ NMR $\left(101 \mathrm{MHz}, \mathrm{DMSO}-d_{6}\right) \delta 175.15,168.22,160.91,155.69$, $143.62,138.76,137.89,130.28,130.28,129.37,129.37$, $124.79,124.05,123.91,123.11,123.11,122.60,122.19$ 119.16, 119.16; HRMS (ESI) calcd for $\mathrm{C}_{20} \mathrm{H}_{11} \mathrm{~N}_{3} \mathrm{O}_{2} \mathrm{~F}_{4} \mathrm{Cl}$ $[\mathrm{M}+\mathrm{H}]^{+}:$436.04312, found 436.04704 .

3-(4-((3-chloro-5-(trifluoromethyl)pyridin-2-yl)oxy) phenyl)-5-(4-methoxyphenyl)-1,2,4-oxadiazole Yellow solid; yield 62.9\%; mp: 95.2-96.0 ${ }^{\circ} \mathrm{C}$; ${ }^{1} \mathrm{H}$ NMR $\left(400 \mathrm{MHz}, \mathrm{DMSO}-d_{6}\right) \delta 8.69$ (d, $J=2.0 \mathrm{~Hz}, 1 \mathrm{H}$, Pyridine$\mathrm{H}), 8.61(\mathrm{~d}, J=1.1 \mathrm{~Hz}, 1 \mathrm{H}$, Pyridine-H), 8.25-8.19 (m, 4H, Ph-H), 7.57-7.53 (m, 2H, Ph-H), 7.27 (d, J=8.9 Hz, 2H, Ph-H), 3.95 (s, 3H, $\left.-\mathrm{OCH}_{3}\right) .{ }^{13} \mathrm{C}$ NMR (101 MHz, DMSO- $\left.d_{6}\right) \delta 175.83,167.99,160.94,155.53,143.62$, $137.88,130.47,130.47,129.31,129.31,124.79,124.21$, 123.07, 123.07, 122.48, 122.15, 119.12, 116.11, 115.51, 115.51, 56.49; HRMS(ESI) calcd for $\mathrm{C}_{21} \mathrm{H}_{14} \mathrm{~N}_{3} \mathrm{O}_{3} \mathrm{~F}_{3} \mathrm{Cl}$; $[\mathrm{M}+\mathrm{H}]^{+}:$448.06703, found 448.06580.

(E)-3-(4-((3-chloro-5-(trifluoromethyl)pyridin-2-yl) oxy)phenyl)-5-styryl-1,2,4-oxadiazole $(\mathbf{5} \mathbf{~ m})$ Light yellow solid; yield 41.7\%; mp: $121.0-121.5{ }^{\circ} \mathrm{C}$; ${ }^{1} \mathrm{H}$ NMR $\left(400 \mathrm{MHz}, \mathrm{DMSO}-d_{6}\right) \delta 8.64(\mathrm{~d}, J=2.1 \mathrm{~Hz}, 1 \mathrm{H}$, Pyridine$\mathrm{H}), 8.56$ (dd, $J=2.1,1.0 \mathrm{~Hz}, 1 \mathrm{H}$, Pyridine-H), 8.14-8.09 (m, 2H, -CH-), 7.98 (d, J=16.4 Hz, 1H, Ph-H), 7.907.86 (m, 2H Ph-H), 7.52-7.46 (m, 6H, Ph-H). ${ }^{13} \mathrm{C}$ NMR $\left(101 \mathrm{MHz}, \mathrm{DMSO}-d_{6}\right) \delta 175.99,167.89,160.93,155.54$, $144.29,143.34,137.88,134.69,131.20,129.51,129.51$, $129.26,129.26,128.92,124.79,124.19,123.07,123.07$, 122.48, 122.12, 119.14, 110.66; HRMS (ESI) calcd for $\mathrm{C}_{22} \mathrm{H}_{14} \mathrm{~N}_{3} \mathrm{O}_{2} \mathrm{~F}_{3} \mathrm{Cl}[\mathrm{M}+\mathrm{H}]^{+}:$444.07135, found 444.07212.

3-(4-((3-chloro-5-(trifluoromethyl)pyridin-2-yl) oxy)phenyl)-5-(4-nitrophenyl)-1,2,4-oxadiazole

(5n) White solid; yield $35.8 \%$; mp: $182.4-183.7{ }^{\circ} \mathrm{C}$; ${ }^{1} \mathrm{H}$ NMR $\left(400 \mathrm{MHz}, \mathrm{DMSO}-d_{6}\right) \delta 8.64(\mathrm{~d}, J=2.1 \mathrm{~Hz}, 1 \mathrm{H}$, Pyridine$\mathrm{H}), 8.65(\mathrm{~d}, J=1.9 \mathrm{~Hz}, 1 \mathrm{H}$, Pyridine-H), $8.49(\mathrm{~d}, J=3.4 \mathrm{~Hz}$, 4H, Ph-H), 8.24-8.19 (m, 2H, Ph-H), 7.56-7.50 (m, 2H, Ph-H). ${ }^{13} \mathrm{C}$ NMR (101 MHz, DMSO- $\left.d_{6}\right) \delta 175.1,168.14$, $160.61,157.93,155.62,143.61,137.82,132.66,131.32$, $129.75,129.33,129.33,124.78,123.90,123.90,122.50$, 121.84, 120.51, 119.14, 117.25; HRMS (ESI) calcd for $\mathrm{C}_{20} \mathrm{H}_{11} \mathrm{~N}_{4} \mathrm{O}_{4} \mathrm{~F}_{3} \mathrm{Cl}[\mathrm{M}+\mathrm{H}]^{+}:$463.04154, found 463.04025. 
3-(4-((3-chloro-5-(trifluoromethyl)pyridin-2-yl)oxy) phenyl)-5-(4-(trifluoromethyl)phenyl)-1,2,4-oxadiazole (5o) White solid; yield 44.3\%; mp: $102.8-103.7{ }^{\circ} \mathrm{C} ;{ }^{1} \mathrm{H}$ NMR $\left(400 \mathrm{MHz}\right.$, DMSO- $\left.d_{6}\right) \delta 8.59(\mathrm{~d}, J=2.2 \mathrm{~Hz}, 1 \mathrm{H}$, Pyridine-H), 8.51 (d, $J=1.0 \mathrm{~Hz}, 1 \mathrm{H}$, Pyridine- $\mathrm{H}), 8.36$ (d, $J=8.3 \mathrm{~Hz}, 2 \mathrm{H}$, Pyridine-H) 8.17-8.13 (m, 2H, Ph-H), 8.00 (d, $J=8.4 \mathrm{~Hz}, 2 \mathrm{H}, \mathrm{Ph}-\mathrm{H}), 7.48-7.45(\mathrm{~m}, 2 \mathrm{H}, \mathrm{Ph}-\mathrm{H})$. ${ }^{13} \mathrm{C}$ NMR $\left(101 \mathrm{MHz}\right.$, DMSO- $\left.d_{6}\right) \delta 174.84,168.40$, $160.95,155.78,143.68,137.93,133.62,133.36,133.11$, $129.87,129.87,128.32,127.61,125.24,124.57,123.81$, $123.81,122.73,122.21,120.00,119.22$; HRMS (ESI) calcd for $\mathrm{C}_{21} \mathrm{H}_{11} \mathrm{~N}_{3} \mathrm{O}_{2} \mathrm{~F}_{6} \mathrm{Cl} ;[\mathrm{M}+\mathrm{H}]^{+}: 486.04385$, found 486.04257.

3-(4-((3-chloro-5-(trifluoromethyl)pyridin-2-yl)oxy) phenyl)-5-isopropyl-1,2,4-oxadiazole (5p) White solid; yield 56.1\%; mp 88.5-89.2 ${ }^{\circ} \mathrm{C}$; ${ }^{1} \mathrm{H}$ NMR $(400 \mathrm{MHz}$, DMSO- $\left.d_{6}\right) \delta 8.63(\mathrm{~d}, J=2.1 \mathrm{~Hz}, 1 \mathrm{H}$, Pyridine- $\mathrm{H})$, 8.55-8.54 (m, $1 \mathrm{H}$, Pyridine-H), $8.09(\mathrm{~d}, J=8.8 \mathrm{~Hz}, 2 \mathrm{H}$, Ph-H), 7.47-7.43 (m, 2H, Ph-H), 2.81 (d, $J=63.7 \mathrm{~Hz}$, $1 \mathrm{H},-\mathrm{CH}-), 1.39$ (d, $\left.J=7.0 \mathrm{~Hz}, 6 \mathrm{H},-\mathrm{CH}_{3}\right) .{ }^{13} \mathrm{C}$ NMR $\left(101 \mathrm{MHz}, \mathrm{DMSO}-d_{6}\right) \delta 184.70,167.30,160.89,155.43$, $143.63,137.83,129.75,129.19,126.50,124.78,124.22$, 123.01, 122.48, 122.11, 119.13, 27.28, 20.29; HRMS (ESI) calcd for $\mathrm{C}_{17} \mathrm{H}_{14} \mathrm{~N}_{3} \mathrm{O}_{2} \mathrm{~F}_{3} \mathrm{Cl}[\mathrm{M}+\mathrm{H}]^{+}$: 384.07212, found 384.07095 .

3-(4-((3-chloro-5-(trifluoromethyl)pyridin-2-yl)oxy) phenyl)-5-ethyl-1,2,4-oxadiazole (5q) White solid; yield 44.8\%; mp 83.3-84.0 ${ }^{\circ} \mathrm{C} ;{ }^{1} \mathrm{H}$ NMR (400 MHz, DMSO- $d_{6}$ ) $\delta 8.63(\mathrm{~d}, J=2.0 \mathrm{~Hz}, 1 \mathrm{H}$, Pyridine- $\mathrm{H}), 8.55(\mathrm{~d}, J=1.1 \mathrm{~Hz}$, $1 \mathrm{H}$, Pyridine-H), 8.09 (d, J=8.7 Hz, 2H, Ph-H), 7.45 (d, $J=8.7 \mathrm{~Hz}, 2 \mathrm{H}, \mathrm{Ph}-\mathrm{H}), 3.03\left(\mathrm{~d}, J=7.6 \mathrm{~Hz}, 2 \mathrm{H},-\mathrm{CH}_{2}-\right.$ ), $1.35\left(\mathrm{t}, J=7.6 \mathrm{~Hz}, 3 \mathrm{H},-\mathrm{CH}_{3}-\right) .{ }^{13} \mathrm{C}$ NMR $(101 \mathrm{MHz}$, DMSO- $\left.d_{6}\right) \delta 181.81,167.34,160.91,155.42,143.62$, $137.84,129.77,129.15,124.79,124.23,123.02,122.46$, 122.10, 119.12, 20.07, 10.92; HRMS (ESI) calcd for $\mathrm{C}_{16} \mathrm{H}_{12} \mathrm{~N}_{3} \mathrm{O}_{2} \mathrm{~F}_{3} \mathrm{Cl}[\mathrm{M}+\mathrm{H}]^{+}: 370.05647$, found 370.05554 .

3-(4-((3-chloro-5-(trifluoromethyl)pyridin-2-yl)oxy) phenyl)-5-(chloromethyl)-1,2,4-oxadiazole (5r) White solid; yield 46.7\%; mp: 94.6-95.7 ${ }^{\circ} \mathrm{C}$; ${ }^{1} \mathrm{H}$ NMR $(500 \mathrm{MHz}$, DMSO- $\left.d_{6}\right) \delta 8.59(\mathrm{~d}, J=2.1 \mathrm{~Hz}, 1 \mathrm{H}$, Pyridine-H), $8.51(\mathrm{dd}$, $J=1.9,0.8 \mathrm{~Hz}, 1 \mathrm{H}$, Pyridine-H), 8.10-8.05 (m, 2H, Ph-H), 7.46-7.42 (m, 2H, Ph-H), 5.17 (s, 2H, $\left.-\mathrm{CH}_{2}-\right) .{ }^{13} \mathrm{C}$ NMR $\left(126 \mathrm{MHz}, \mathrm{DMSO}-d_{6}\right) \delta 176.24,167.98,160.93,155.82$, $143.69,138.04,137.82,129.39,124.57,123.58,123.41$, $122.05,120.00,119.23,34.26$; HRMS (ESI) calcd for $\mathrm{C}_{15} \mathrm{H}_{9} \mathrm{~N}_{3} \mathrm{O}_{2} \mathrm{~F}_{3} \mathrm{Cl}_{2}[\mathrm{M}+\mathrm{H}]^{+}: 390.00184$, found 390.00082 .

3-(4-((3-chloro-5-(trifluoromethyl)pyridin-2-yl)oxy) phenyl)-5-((2,4-dichlorophenoxy)methyl)-1,2,4-oxadiazole $(5 \mathrm{~s})$ Yellow solid; yield $49.0 \%$; mp: $97.1-98.0{ }^{\circ} \mathrm{C}$; ${ }^{1} \mathrm{H}$ NMR $\left(400 \mathrm{MHz}, \mathrm{DMSO}-d_{6}\right) \delta 8.59(\mathrm{~d}, J=2.2 \mathrm{~Hz}$, $1 \mathrm{H}$, Pyridine- $\mathrm{H}), 8.50(\mathrm{~s}, 1 \mathrm{H}$, Pyridine- $\mathrm{H}), 8.06$ (d, $J=8.7 \mathrm{~Hz}, 2 \mathrm{H}, \mathrm{Ph}-\mathrm{H}), 7.62(\mathrm{~d}, J=2.5 \mathrm{~Hz}, 1 \mathrm{H}, \mathrm{Ph}-\mathrm{H})$,
7.45-7.42 (m, 2H, Ph-H), $7.40(\mathrm{dd}, J=8.9,2.6 \mathrm{~Hz}$, $1 \mathrm{H}, \mathrm{Ph}-\mathrm{H}), 7.32(\mathrm{~d}, J=9.0 \mathrm{~Hz}, 1 \mathrm{H}, \mathrm{Ph}-\mathrm{H}), 5.72(\mathrm{~s}, 2 \mathrm{H}$, $\left.-\mathrm{CH}_{2}-\right) .{ }^{13} \mathrm{C}$ NMR (101 MHz, DMSO- $\left.d_{6}\right) \delta$ 175.82, $167.71,160.94,155.77,152.45,152.27,143.67,137.92$, $130.19,130.19,128.78,126.52,124.57,123.64,123.63$, 123.63, 122.67, 122.20, 119.21, 116.34, 62.50; HRMS (ESI) calcd for $\mathrm{C}_{21} \mathrm{H}_{12} \mathrm{~N}_{3} \mathrm{O}_{3} \mathrm{~F}_{3} \mathrm{Cl}_{3}[\mathrm{M}+\mathrm{H}]^{+}$: 515.98779, found 515.98909 .

4-((3-(4-((3-chloro-5-(trifluoromethyl)pyridin-2-yl) oxy)phenyl)-1,2,4-oxadiazol-5-yl)methoxy)benzonitrile (5t) White solid; yield 61.8\%; mp: $119.0-120.2{ }^{\circ} \mathrm{C} ;{ }^{1} \mathrm{H}$ NMR $\left(400 \mathrm{MHz}\right.$, DMSO- $\left.d_{6}\right) \delta: 8.59(\mathrm{~d}, J=2.1 \mathrm{~Hz}, 1 \mathrm{H}$, Pyridine- $\mathrm{H}), 8.50(\mathrm{~d}, J=1.1 \mathrm{~Hz}, 1 \mathrm{H}$, Pyridine- $\mathrm{H}), 8.06(\mathrm{~d}$, $J=8.7 \mathrm{~Hz}, 2 \mathrm{H}, \mathrm{Ph}-\mathrm{H}), 7.82(\mathrm{~d}, J=8.9 \mathrm{~Hz}, 2 \mathrm{H}, \mathrm{Ph}-\mathrm{H}), 7.44$ $(\mathrm{d}, J=8.7 \mathrm{~Hz}, 2 \mathrm{H}, \mathrm{Ph}-\mathrm{H}), 7.26$ (d, $J=8.9 \mathrm{~Hz}, 2 \mathrm{H}, \mathrm{Ph}-\mathrm{H})$, $5.72\left(\mathrm{~s}, 2 \mathrm{H},-\mathrm{CH}_{2}-\right) .{ }^{13} \mathrm{C}$ NMR (101 MHz, DMSO- $\left.d_{6}\right) \delta$ $175.81,167.72,160.93,155.78,143.68,137.95,134.94$, $134.94,129.42,129.42,124.57,123.62,123.22,123.22$, $122.63,122.21,119.39,119.22,116.54,116.36,104.80$, 61.61; HRMS (ESI) calcd for $\mathrm{C}_{22} \mathrm{H}_{13} \mathrm{~N}_{4} \mathrm{O}_{3} \mathrm{~F}_{3} \mathrm{Cl}[\mathrm{M}+\mathrm{H}]^{+}$: 473.06228, found 473.06107.

3-(4-((3-chloro-5-(trifluoromethyl)pyridin-2-yl)oxy) phenyl)-5-((4-fluorophenoxy)methyl)-1,2,4-oxadiazole (5u) Yellow solid; yield 41.8\%; mp: $101.1-102.0{ }^{\circ} \mathrm{C} ;{ }^{1} \mathrm{H}$ NMR $\left(400 \mathrm{MHz}\right.$, DMSO- $\left.d_{6}\right) \delta 8.63(\mathrm{~d}, J=2.1 \mathrm{~Hz}, 1 \mathrm{H}$, Pyridine-H), 8.55-8.53 (m, 1H, Pyridine-H), 8.13-8.09 (m, 2H, Ph-H), 7.49-7.45 (m, 2H, Ph-H), 7.21-7.11 (m, $4 \mathrm{H}, \mathrm{Ph}-\mathrm{H}), 5.60\left(\mathrm{~s}, 2 \mathrm{H},-\mathrm{CH}_{2}-\right) .{ }^{13} \mathrm{C}$ NMR $(101 \mathrm{MHz}$, DMSO- $\left.d_{6}\right) \delta 176.30,167.61,160.88,158.86,156.50$, $155.69,154.06,143.61,137.85,129.34,129.34,124.78$, $123.65,123.14,123.14,122.52,122.13,119.16,116.99$, 116.99, 61.91; HRMS (ESI) calcd for $\mathrm{C}_{21} \mathrm{H}_{13} \mathrm{~N}_{3} \mathrm{O}_{3} \mathrm{~F}_{4} \mathrm{Cl}$ $[\mathrm{M}+\mathrm{H}]^{+}:$: 466.05761, found 466.05652 .

3-(4-((3-chloro-5-(trifluoromethyl)pyridin-2-yl)oxy) phenyl)-5-((4-chlorophenoxy)methyl)-1,2,4-oxadiazole (5v) Brown solid; yield 48.5\%; mp: $92.3-93.1{ }^{\circ} \mathrm{C} ;{ }^{1} \mathrm{H}$ NMR $\left(400 \mathrm{MHz}, \mathrm{DMSO}-d_{6}\right) \delta 8.64(\mathrm{~d}, J=2.0 \mathrm{~Hz}, 1 \mathrm{H}$, Pyridine$\mathrm{H}), 8.55$ (dd, $J=2.1,0.9 \mathrm{~Hz}, 1 \mathrm{H}$, Pyridine-H), 8.12-8.09 (m, 2H, Ph-H), 7.49-7.46 (m, 2H, Ph-H), 7.42-7.39 (m, $2 \mathrm{H}, \mathrm{Ph}-\mathrm{H}), 7.16-7.13(\mathrm{~m}, 2 \mathrm{H}, \mathrm{Ph}-\mathrm{H}), 5.64\left(\mathrm{~s}, 2 \mathrm{H},-\mathrm{CH}_{2}-\right.$ ). ${ }^{13} \mathrm{C}$ NMR $\left(101 \mathrm{MHz}, \mathrm{DMSO}-d_{6}\right) \delta 176.16,167.62,160.88$, $156.59,155.70,143.63,137.87,129.95,129.95,129.35$, $126.11,124.79,123.62,123.17,123.17,122.50,122.13$, 119.15, 117.13, 117.13, 61.59; HRMS (ESI) calcd for $\mathrm{C}_{21} \mathrm{H}_{13} \mathrm{~N}_{3} \mathrm{O}_{3} \mathrm{~F}_{3} \mathrm{Cl}_{2}[\mathrm{M}+\mathrm{H}]^{+}:$482.02806, found 482.02698.

3-(4-((3-chloro-5-(trifluoromethyl)pyridin-2-yl)oxy) phenyl)-5-((p-tolyloxy)methyl)-1,2,4-oxadiazole (5w) Yellow solid; yield 54.9\%; mp: 84.2-84.7 ${ }^{\circ} \mathrm{C}$; ${ }^{1} \mathrm{H}$ NMR $\left(500 \mathrm{MHz}\right.$, DMSO- $\left.d_{6}\right) \delta 8.59(\mathrm{~d}, J=2.1 \mathrm{~Hz}, 1 \mathrm{H}, \mathrm{Pyr}-$ idine-H), 8.50 (dd, $J=1.9,0.8 \mathrm{~Hz}, 1 \mathrm{H}$, Pyridine- $\mathrm{H})$, 8.09-8.05 (m, 2H, Ph-H), 7.45-7.41 (m, 2H, Ph-H), 7.10 (d, J=8.2 Hz, 2H, Ph-H), 6.96-6.93 (m, 2H, Ph-H), $5.53\left(\mathrm{~s}, 2 \mathrm{H},-\mathrm{CH}_{2}-\right), 2.20\left(\mathrm{~s}, 3 \mathrm{H},-\mathrm{CH}_{3}\right) .{ }^{13} \mathrm{C} \mathrm{NMR}$ 
(126 MHz, DMSO- $\left.d_{6}\right) \delta 176.62,167.65,160.94,155.72$, $143.68,137.94,131.22,130.55,130.55,129.40,129.40$, $124.57,123.74,123.21,123.21,122.62,122.2,119.22$, 115.18, 115.18, 61.39, 20.61; HRMS (ESI) calcd for $\mathrm{C}_{22} \mathrm{H}_{16} \mathrm{~N}_{3} \mathrm{O}_{3} \mathrm{~F}_{3} \mathrm{Cl} ;[\mathrm{M}+\mathrm{H}]^{+}:$462.08268, found 462.08160 .

4-(4-((3-chloro-5-(trifluoromethyl)pyridin-2-yl)oxy) phenyl)-5-((o-tolyloxy)methyl)-1,2,4-oxadiazole (5x) Yellow solid; yield 42.2\%; mp: 80.0-80.8 ${ }^{\circ} \mathrm{C}$; ${ }^{1} \mathrm{H}$ NMR $\left(500 \mathrm{MHz}, \mathrm{DMSO}-d_{6}\right) \delta 8.60(\mathrm{~d}, J=2.0 \mathrm{~Hz}, 1 \mathrm{H}$, Pyridine-H), 8.51 (s, $1 \mathrm{H}$, Pyridine- $\mathrm{H}), 7.44(\mathrm{~d}, J=8.7 \mathrm{~Hz}$, $3 \mathrm{H}, \mathrm{Ph}-\mathrm{H}), 7.16$ (dd, J=13.7, 7.4 Hz, $2 \mathrm{H}, \mathrm{Ph}-\mathrm{H}), 7.05$ (d, $J=8.0 \mathrm{~Hz}, 1 \mathrm{H}, \mathrm{Ph}-\mathrm{H}), 6.89$ (t, $J=7.3 \mathrm{~Hz}, 1 \mathrm{H}, \mathrm{Ph}-\mathrm{H})$, $5.59\left(\mathrm{~s}, 2 \mathrm{H},-\mathrm{CH}_{2}-\right), 2.20\left(\mathrm{~s}, 3 \mathrm{H},-\mathrm{CH}_{3}\right),{ }^{13} \mathrm{C} \mathrm{NMR}$ $\left(126 \mathrm{MHz}, \mathrm{DMSO}-d_{6}\right) \delta 176.70,167.67,160.95,155.98$, $155.75,143.69,137.95,131.37,129.40,129.40,127.62$, $126.80,124.58,123.75,123.23,123.23,122.67,121.90$, 119.21, 112.57, 61.68, 16.45; HRMS (ESI) calcd for $\mathrm{C}_{22} \mathrm{H}_{16} \mathrm{~N}_{3} \mathrm{O}_{3} \mathrm{~F}_{3} \mathrm{Cl}[\mathrm{M}+\mathrm{H}]^{+}: 462.08268$, found 462.08160 .

\section{Nematocidal activity}

The nematocidal activity of the target compound was carried out according to the reported method [33]. The tomato grown in the greenhouse for cultivating southern root-knot nematodes were uprooted and washed with water. Then take the eggs of the roots with a toothpick and place them in a petri dish containing distilled water. Second instar larvae were collected after 3-7 days of incubation at $27^{\circ} \mathrm{C}$. All tested compounds were dissolved in DMF and diluted with $1 \%$ Tween 80 (final concentration of DMF was $0.5 \%$ ). $250 \mu \mathrm{L}$ of the test solution was added to a 48-well biochemical culture dish and tested. Subsequently, approximately 100 nematodes were added to each well. Abamectin was used as a positive control, and a test solution containing no compound was used as a negative control. After $48 \mathrm{~h}$ of treatment with the compound, the nematode was transferred to clear water for resuscitation, and the nematode that did not move was considered dead.

Corrected mortality $\%=[($ mortality of treatment $\%-$ mortality of negative control $\%)$

$/(1-$ mortality of negative control $\%)] \times 100$

4-((3-(4-((3-chloro-5-(trifluoromethyl)pyridin-2-yl)oxy) phenyl)-1,2,4-oxadiazol-5-yl)methoxy)-2-fluorobenzonitrile (5y) Red solid; yield 43.7\%; mp: $114.7-115.3{ }^{\circ} \mathrm{C} ;{ }^{1} \mathrm{H}$ NMR (400 MHz, DMSO- $\left.d_{6}\right) \delta 8.59$ (d, $J=2.2 \mathrm{~Hz}, 1 \mathrm{H}$, Pyridine-H), 8.50 (d, $J=1.0 \mathrm{~Hz}, 1 \mathrm{H}$, Pyridine- $\mathrm{H}), 8.08-$ 8.04 (m, 2H, Ph-H), 7.92-7.87 (m, 1H, Ph-H), 7.45-7.42 (m, 2H, Ph-H), 7.38 (dd, $J=11.6,2.4 \mathrm{~Hz}, 1 \mathrm{H}, \mathrm{Ph}-\mathrm{H}), 7.14$ (dd, $J=8.8,2.4 \mathrm{~Hz}, 1 \mathrm{H}, \mathrm{Ph}-\mathrm{H}), 5.75\left(\mathrm{~s}, 2 \mathrm{H},-\mathrm{CH}_{2}-\right) .{ }^{13} \mathrm{C}$ NMR (101 MHz, DMSO- $d_{6}$ ) $\delta$ 175.34, 167.65, 160.87, $155.72,143.61,137.87,135.49,129.36,129.36,124.77$, $123.58,123.35,123.35,122.07,119.15,114.63,113.14$, 104.17, 104.03, 103.59, 93.50, 62.12; HRMS (ESI) calcd for $\mathrm{C}_{22} \mathrm{H}_{12} \mathrm{~N}_{4} \mathrm{O}_{3} \mathrm{~F}_{4} \mathrm{Cl}[\mathrm{M}+\mathrm{H}]^{+}$: 491.05286, found 491.05167.

3-(4-((3-chloro-5-(trifluoromethyl)pyridin-2-yl)oxy) phenyl)-5-ethyl-1,2,4-oxadiazole (5z) White solid; yield 57.5\%; mp: $105.2-106.7{ }^{\circ} \mathrm{C}$; ${ }^{1} \mathrm{H}$ NMR $(400 \mathrm{MHz}$, DMSO- $\left.d_{6}\right) \delta 8.64(\mathrm{~d}, J=2.0 \mathrm{~Hz}, 1 \mathrm{H}$, Pyridine- $\mathrm{H}), 8.55$ (d, $J=1.1 \mathrm{~Hz}, 1 \mathrm{H}$, Pyridine- $\mathrm{H}), 8.54(\mathrm{dd}, J=2.1,0.9 \mathrm{~Hz}$, 2H, Ph-H), 8.13-8.09 (m, 2H, Ph-H), 7.50-7.45 (m, 2H, $\mathrm{Ph}-\mathrm{H}), 7.25-7.19$ (m, 2H, Ph-H), 5.67 (s, $2 \mathrm{H},-\mathrm{CH}_{2}-$ ). ${ }^{13} \mathrm{C}$ NMR $\left(101 \mathrm{MHz}, \mathrm{DMSO}-d_{6}\right) \delta 176.12,167.63,160.88$, $156.57,155.70,143.62,143.11,137.89,137.87,129.36$, $129.36,124.78,123.62,123.20,123.20,122.51,122.13$, 121.87, 119.24, 116.68, 116.68, 61.75; HRMS (ESI) calcd for $\mathrm{C}_{22} \mathrm{H}_{13} \mathrm{~N}_{3} \mathrm{O}_{4} \mathrm{~F}_{6} \mathrm{Cl}[\mathrm{M}+\mathrm{H}]^{+}:$532.04933, found 532.04822 .

\section{Antifungal assay}

The mycelium growth rate method was utilized to evaluate in vitro antifungal activities of target compounds against $R$. solani [34]. The DMSO solution of the test compound was added to a sterilized petri dish containing about $10 \mathrm{~mL}$ of molten potato dextrose agar (PDA). Subsequently, a mycelial plug with a diameter of $4 \mathrm{~mm}$ was cut from the fungal colony and placed in the center of the PDA plate at $28 \pm 1{ }^{\circ} \mathrm{C}$ for 4 days. For each compound, antifungal assays were performed in triplicate. In addition, pure DMSO and commercial fungicide (Hymexazol) were also used as negative and positive control agents, respectively.

The inhibition rate $(I)$ of the tested compound was determined based on the following formula:

$$
I=(\mathrm{C}-\mathrm{T}) /(\mathrm{C}-0.4) \times 100 \%
$$

In the formula, $\mathrm{C}$ represents the average mycelial diameter of negative control and $\mathrm{T}$ represents the average mycelial diameter of tested compound-treated PDA.

\section{Antibacterial activity in vitro}

The previously described method was used for in vitro antibacterial activity testing [35-37]. The $50 \mu \mathrm{L}$ culture of Xoo or Xoc in logarithmic growth phase were added to the test tubes with $5 \mathrm{~mL}$ of NB medium containing different concentrations of the target compound, respectively. The commercial bacteriacide thiodiazole copper 
(TDC, 20\% suspending agent) and bismerthiazol (BMT, $20 \%$ wettable powder) as positive controls, while the same treatment without compound as the negative control. Then, the absorbance at $600 \mathrm{~nm}$ was measured when the tubes were inoculated at $28^{\circ} \mathrm{C}$ for $48 \mathrm{~h}$ with shaking at $180 \mathrm{rpm}$. The inhibition rate (the inhibition rate refers to the proportion of bacteria whose growth was inhibited) was calculated by the following equation: where the absorbance of the negative control group was expressed as $\mathrm{OD}_{\mathrm{CK}}$, and the absorbance of the treated group was expressed as $\mathrm{OD}_{\mathrm{T}}$.

$$
\text { Inhibition rate }(\%)=\left(O D_{\mathrm{CK}}-O D_{\mathrm{T}}\right) / O D_{\mathrm{CK}} \times 100
$$

\section{Antibacterial activity in vivo}

In vivo biometric against rice bacterial leaf blight. The curative and protection activities of compound $\mathbf{5 v}$ against rice bacterial leaf blight were determined by Schaad's method with some slight modifications [4]. The curative activity of the rice plant bacterial leaf blight-reducing compound $5 \mathbf{v}$ in potted plants was determined under controlled conditions in the growth room. About 8 weeks after planting the "Fengyouxiangzhan" rice seeds, Xoo was inoculated on the rice leaves. One day after the inoculation, $200 \mu \mathrm{g} / \mathrm{mL} \mathbf{5 v}$ solutions was evenly sprayed onto the rice leaves until dripping, and $200 \mu \mathrm{g} / \mathrm{mL}$ BMT and TDC solutions, and distilled water was evenly sprayed as positive and negative control groups, respectively. Then, all inoculated rice plants were placed in a plant growth chamber $\left(28{ }^{\circ} \mathrm{C}\right.$ and $90 \%$ relative humidity). On the 14th day after spraying, the disease index of the inoculated rice leaves was measured. Similarly, the protective activity of reducing rice bacterial leaf blight of compound $5 \mathbf{v}$ was also evaluated, the difference is that 1 day after spraying the compound solution and distilled water, Xoo was inoculated on rice leaves and the disease index of the inoculated rice leaves was measured on the 14th day after inoculation. First, measure the spot area of each leaf and the entire leaf area, and then calculate the percentage of the spot area in the entire leaf area. Secondly, these leaves were classed according to the following grading standards. Grade 1: the area of disease spot accounts for less than $5 \%$ of the whole leaf area; Grade 3: the area of disease spot accounts for $6-10 \%$ of the whole leaf area; Grade 5: the area of disease spot accounts for $11-20 \%$ of the whole leaf area; Grade 7: the area of disease spot 6 accounts for $21-50 \%$ of the whole leaf area; Grade 9: the area of disease spot accounts for more than $50 \%$ of the whole leaf area. Finally, the disease index $(\mathrm{C}$ or $\mathrm{T})$ was calculated using the following formula: Disease index $(\mathrm{C}$ or $\mathrm{T})=\sum$ (the number of leaves at each Grade $\times$ the corresponding Grade)/(the total number of leaves $\times$ the superlative Grade). The control coefficients
I (\%) for the curative and protection activities are calculated by the following equation. In the equation, $\mathrm{C}$ is the disease index of the negative control and $\mathrm{T}$ is the disease index of the treatment group.

$$
\text { Control efficiency } \mathrm{I}(\%)=(\mathrm{C}-\mathrm{T}) / \mathrm{C} \times 100
$$

\section{Results and discussion}

\section{Design of novel 1,2,4-oxadiazole derivatives}

1,2,4-Oxadiazole heterocycle is an important pharmacophore to design novel drug molecules. The compounds containing 1,2,4-oxadiazole skeleton possess various bioactivity in agricultural, including antibacterial, antifungal and nematocidal activities. Of which, tioxazafen is a new nematicide with unique mechanism of action developed by Monsanto. In our previous works, some 1,3,4-thiadiazol or 1,3,4-oxadiazole derivatives were designed and synthesized, and they exhibited good antibacterial, antifungal and nematocidal activities. So, we firstly introduced 1,3,4-thiadiazol or 1,3,4-oxadiazole into 1,2,4-oxadiazole skeleton to find highly active compounds. Meanwhile, the literature survey reveals that fluopyram showed good antibacterial activity and nematocidal activity, and the important pharmacophore is trifluoromethyl pyridine moiety. Encouraged by this results, we designed the novel 1,2,4-oxadiazole derivatives containing a trifluoromethyl pyridine moiety to find new lead compounds.

\section{Chemistry}

${ }^{1} \mathrm{H}$ NMR, ${ }^{13} \mathrm{C}$ NMR, and HRMS were used to characterize the physical properties of the target compounds 5a-5z. ${ }^{1} \mathrm{H}$ NMR, ${ }^{13} \mathrm{C}$ NMR, and HRMS data are provided in Additional file 1. In ${ }^{1} \mathrm{H}$ NMR spectra of compound $5 \mathbf{v}$, singlet at $\delta 8.64-8.55 \mathrm{ppm}$ reveals the presence of Pyridine-H protons, singlet at $\delta 8.12-7.13 \mathrm{ppm}$ reveals the presence of $\mathrm{Ph}-\mathrm{H}$ protons. From the analysis of the ${ }^{13} \mathrm{C}$ NMR spectrum of the compound $5 \mathbf{v}$, it can be seen that 176.16 and $167.62 \mathrm{ppm}$ are the absorption peaks of carbon on oxadiazole structure, $160.88,156.59$ and $155.70 \mathrm{ppm}$ are the absorption peaks of carbon on the benzene ring directly connected to the oxygen group, and $61.59 \mathrm{ppm}$ is the absorption peak of methylene carbon.

\section{Nematocidal activity screening of title compounds}

The in vitro nematocidal activity of the target compounds 5a-5i was evaluated using the direct strike method against Meloidogyne incognita. The results showed that all of the 1,2,4-oxadiazole derivatives containing 1,3,4-thiadiazol or 1,3,4-oxadiazole moiety have 
low nematocidal activities. And then, introducing the trifluoromethyl pyridine moiety can enhance the activity. Of which, compounds $\mathbf{5 n}$ and $\mathbf{5 v}$ exhibited significant nematocidal activity against $M$. incognita, with the inhibitory ratio of $63.3 \%$ and $55.0 \%$ at $100 \mu \mathrm{g} / \mathrm{mL}$, respectively, which was superior to that of tioxazafen (29.0\%). There was no good activity of the 1,2,4-oxadiazole derivatives containing trifluoromethyl pyridine and diether groups (Table 1).

\section{Antifungal activity screening of title compounds}

Antifungal activity of target compounds was evaluated by using the mycelium growth method. Unfortunately, the results revealed that almost all the compounds failed to exhibit a noticeable fungicidal activity $(\geq 50.0 \%)$ against $R$. solani at $50 \mu \mathrm{g} / \mathrm{mL}$ (Table 1 ).

\section{Antibacterial activity screening of title compounds}

In vitro bacterial activity test was performed using the turbidity method and the results were listed in Table 2 . As shown in Table 2, thioether derivatives containing an 1,2,4-oxadiazole scaffold have low antibacterial activities. Some target compounds introducing the trifluoromethyl pyridine moiety showed better antibacterial activities against $X o o$ and $X o c$ at a concentration of $50 \mu \mathrm{g} / \mathrm{mL}$ compared to the control drugs, for example compounds $\mathbf{5 m}, \mathbf{5 r}, \mathbf{5 u}, \mathbf{5 v}, \mathbf{5 x}$, and $\mathbf{5 y}$ with the values of $65.85,71.89,64.97,85.37,61.97$, and $78.44 \%$ against Xoo, respectively. Meanwhile, Compounds $\mathbf{5 p}$, 5u, and $5 \mathbf{v}$ with the values of $64.53,65.10$, and $64.59 \%$ against Xoo, respectively. The half maximal effective concentration $\left(E_{50}\right)$ value of compounds was further tested. The results clearly showed that some target compounds exhibit better antibacterial activity than that of bismerthiazol (BMT) and thiodiazole copper (TDC). Compounds $\mathbf{5 m}, \mathbf{5 r}, \mathbf{5 u}, \mathbf{5 v}, \mathbf{5 x}$ and $\mathbf{5 y}$ showed excellent antibacterial effects on Xoo, with $\mathrm{EC}_{50}$ values of 36.25 , 24.14, 28.82, 19.44, 25.37 and $28.52 \mu \mathrm{g} / \mathrm{mL}$, respectively, stronger than BMT $\left(\mathrm{EC}_{50}=77.46 \mu \mathrm{g} / \mathrm{mL}\right)$ and $\mathrm{TDC}$ $\left(\mathrm{EC}_{50}=99.31 \mu \mathrm{g} / \mathrm{mL}\right)$. Compounds 5n, 5p, 5t, 5u, 5v and $\mathbf{5 z}$ exhibited strong antibacterial ability against $X o c$, with $\mathrm{EC}_{50}$ values of 50.93, 31.40, 56.50, 19.04, 21.78, and $55.32 \mu \mathrm{g} / \mathrm{mL}$, respectively, superior to the control agents BMT $\left(\mathrm{EC}_{50}=68.50 \mu \mathrm{g} / \mathrm{mL}\right)$ and TDC $\left(\mathrm{EC}_{50}=91.05 \mu \mathrm{g} /\right.$ $\mathrm{mL}$ ). Among these target compounds, compound $5 \mathbf{v}$ and $5 \mathbf{u}$ showed the best antibacterial activity on Xoo and Xoc, respectively.
Compound 5v exhibited excellent antibacterial ability to Xoo, with an $\mathrm{EC}_{50}$ value of $19.44 \mu \mathrm{g} / \mathrm{mL}$. Accordingly, the control effect of compound $5 \mathbf{v}$ on rice bacterial leaf blight was evaluated and the results were showed in Table 3 and Fig. 2. Compound 5v exerted moderate control effects on rice bacterial leaf blight at $200 \mu \mathrm{g} /$ $\mathrm{mL}$, with curative and protective activity values of $37.8 \%$

\begin{tabular}{|c|c|c|}
\hline \multirow[t]{2}{*}{ Compounds } & \multirow{2}{*}{$\begin{array}{l}\text { M. incognita } \\
\text { Corrected mortality } \\
\text { rate }(\%)^{\mathrm{a}} \text {. }\end{array}$} & \multirow{2}{*}{$\begin{array}{l}\text { R. solani } \\
\text { Inhibition rate (\%) }\end{array}$} \\
\hline & & \\
\hline $5 a$ & $33.4 \pm 2.7$ & $28.7 \pm 1.3$ \\
\hline $5 b$ & $28.9 \pm 5.2$ & $15.6 \pm 0.7$ \\
\hline $5 c$ & $37.6 \pm 8.1$ & $25.2 \pm 2.2$ \\
\hline $5 d$ & $26.6 \pm 2.0$ & $20.4 \pm 1.2$ \\
\hline $5 e$ & 0 & $18.8 \pm 1.7$ \\
\hline $5 f$ & $29.5 \pm 2.7$ & $34.6 \pm 2.3$ \\
\hline $5 \mathrm{~g}$ & $31.3 \pm 5.3$ & $16.1 \pm 1.1$ \\
\hline $5 \mathrm{~h}$ & $29.4 \pm 6.1$ & $19.1 \pm 0.5$ \\
\hline $5 i$ & $40.3 \pm 1.8$ & $23.5 \pm 1.6$ \\
\hline $5 j$ & $39.7 \pm 4.2$ & $15.0 \pm 2.3$ \\
\hline $5 \mathrm{k}$ & $14.7 \pm 27$ & $34.7 \pm 0.8$ \\
\hline 51 & $31.3 \pm 3.8$ & $22.1 \pm 0.8$ \\
\hline $5 \mathrm{~m}$ & $48.0 \pm 3.7$ & $23.8 \pm 3.2$ \\
\hline $5 n$ & $63.3 \pm 7.7$ & $29.4 \pm 1.0$ \\
\hline 50 & $35.5 \pm 2.4$ & $47.0 \pm 1.7$ \\
\hline $5 p$ & $25.3 \pm 3.7$ & $10.4 \pm 0.6$ \\
\hline $5 q$ & $44.8 \pm 6.7$ & $26.1 \pm 0.8$ \\
\hline $5 r$ & $36.7 \pm 5.0$ & $29.1 \pm 0.5$ \\
\hline $5 \mathrm{~s}$ & $27.3 \pm 8.8$ & $13.3 \pm 1.6$ \\
\hline $5 t$ & $35.6 \pm 3.4$ & $25.8 \pm 1.3$ \\
\hline $5 u$ & $40.8 \pm 3.4$ & $34.5 \pm 2.3$ \\
\hline $5 v$ & $55.0 \pm 7.7$ & $34.3 \pm 0.3$ \\
\hline $5 w$ & $26.6 \pm 4.0$ & $32.9 \pm 1.1$ \\
\hline $5 x$ & $47.3 \pm 3.8$ & $13.6 \pm 3.2$ \\
\hline $5 y$ & $34.6 \pm 2.4$ & $39.4 \pm 1.4$ \\
\hline $5 z$ & $36.5 \pm 2.6$ & $27.8 \pm 1.6$ \\
\hline Tioxazafen & $29.0 \pm 4.5$ & NT \\
\hline Fluopicolide & 100 & NT \\
\hline Azoxystrobin & NT & 100 \\
\hline
\end{tabular}

Average of three replicates

NT not tested

a Target compounds $\mathbf{5 a - 5 z}$ at a concentration of $100 \mu \mathrm{g} / \mathrm{mL}$ against $J 2$ of $M$. incognita

b Target compounds $\mathbf{5 a}-\mathbf{5 z}$ at a concentration of $50 \mu \mathrm{g} / \mathrm{mL}$ against $R$.solani 
Table 2 Antibacterial activity of the target compounds $5 a-5 z$ against $X o 0$ and $X o c$

\begin{tabular}{|c|c|c|c|c|}
\hline \multirow[t]{2}{*}{ Compounds } & \multicolumn{2}{|l|}{ Xoo } & \multicolumn{2}{|l|}{ Xoc } \\
\hline & $\begin{array}{l}50 \mu \mathrm{g} / \mathrm{mL} \\
(\%)\end{array}$ & $\mathrm{EC}_{50}(\mu \mathrm{g} / \mathrm{mL})$ & $\begin{array}{l}50 \mu \mathrm{g} / \mathrm{mL} \\
(\%)\end{array}$ & $\mathrm{EC}_{50}(\mu \mathrm{g} / \mathrm{mL})$ \\
\hline $5 a$ & $13.50 \pm 3.33$ & NT & $17.20 \pm 1.60$ & NT \\
\hline $5 b$ & 0 & NT & 0 & NT \\
\hline $5 c$ & $29.00 \pm 3.90$ & NT & $20.40 \pm 1.00$ & NT \\
\hline $5 d$ & 0 & NT & 0 & NT \\
\hline $5 e$ & 0 & NT & 0 & NT \\
\hline $5 f$ & $6.70 \pm 4.30$ & NT & $12.60 \pm 1.10$ & NT \\
\hline $5 \mathrm{~g}$ & 0 & NT & 0 & NT \\
\hline $5 \mathrm{~h}$ & $10.10 \pm 3.20$ & NT & $11.30 \pm 5.30$ & NT \\
\hline $5 i$ & $10.90 \pm 1.00$ & NT & $19.40 \pm 2.10$ & NT \\
\hline $5 j$ & $42.17 \pm 1.39$ & $74.70 \pm 5.46$ & $41.37 \pm 5.42$ & $88.47 \pm 4.21$ \\
\hline $5 \mathrm{k}$ & $30.59 \pm 3.19$ & $85.30 \pm 3.66$ & $15.58 \pm 2.84$ & $199.65 \pm 18.98$ \\
\hline 51 & $14.16 \pm 2.93$ & $129.15 \pm 11.16$ & $29.95 \pm 3.57$ & $172.18 \pm 13.94$ \\
\hline $5 \mathrm{~m}$ & $65.85 \pm 3.32$ & $36.25 \pm 1.77$ & $33.01 \pm 3.23$ & $76.35 \pm 4.82$ \\
\hline $5 n$ & $42.77 \pm 2.13$ & $56.75 \pm 5.98$ & $53.73 \pm 3.24$ & $50.93 \pm 8.07$ \\
\hline 50 & $26.94 \pm 0.65$ & $122.80 \pm 7.32$ & $31.60 \pm 5.28$ & $108.27 \pm 5.99$ \\
\hline $5 p$ & $11.99 \pm 1.18$ & $225.35 \pm 8.03$ & $64.53 \pm 3.42$ & $31.40 \pm 2.07$ \\
\hline $5 q$ & $43.78 \pm 2.02$ & $63.32 \pm 1.58$ & $33.61 \pm 4.13$ & $123.34 \pm 8.55$ \\
\hline $5 r$ & $71.89 \pm 1.56$ & $24.14 \pm 2.72$ & $48.53 \pm 1.66$ & $51.11 \pm 3.05$ \\
\hline $5 \mathrm{~s}$ & $26.30 \pm 2.37$ & $95.12 \pm 14.87$ & $30.28 \pm 2.88$ & $116.76 \pm 13.97$ \\
\hline $5 t$ & $38.16 \pm 2.27$ & $102.45 \pm 5.99$ & $50.37 \pm 3.23$ & $56.50 \pm 6.26$ \\
\hline $5 u$ & $64.97 \pm 3.46$ & $28.82 \pm 2.25$ & $65.10 \pm 4.12$ & $19.04 \pm 2.13$ \\
\hline $5 v$ & $85.37 \pm 1.46$ & $19.44 \pm 2.22$ & $64.59 \pm 4.43$ & $21.78 \pm 2.82$ \\
\hline $5 w$ & $40.60 \pm 3.95$ & $75.61 \pm 2.21$ & $40.12 \pm 3.23$ & $75.22 \pm 3.93$ \\
\hline $5 x$ & $61.97 \pm 0.92$ & $25.37 \pm 6.98$ & $40.29 \pm 3.84$ & $101.96 \pm 5.81$ \\
\hline $5 y$ & $78.44 \pm 5.54$ & $28.52 \pm 3.14$ & $20.63 \pm 6.75$ & $125.57 \pm 15.77$ \\
\hline $5 z$ & $44.07 \pm 2.36$ & $66.51 \pm 5.53$ & $53.70 \pm 3.73$ & $55.32 \pm 2.96$ \\
\hline BMT & $35.12 \pm 2.72$ & $77.46 \pm 6.15$ & $45.56 \pm 5.76$ & $68.50 \pm 7.87$ \\
\hline TDC & $25.12 \pm 3.31$ & $99.31 \pm 7.59$ & $37.58 \pm 2.84$ & $91.05 \pm 8.84$ \\
\hline
\end{tabular}

Average of three replicates

$N T$ not tested and $27.6 \%$, respectively, lower than those of the control agents BMT (46.7\% and 36.1\%, respectively) and TDC (31.8\% and $30.9 \%$, respectively).

The preliminary structure-activity relationship analysis results were as follows: Firstly, the introduction of strong electron withdrawing groups, such as $-\mathrm{NO}_{2}(\mathbf{5 n}$, $\left.\mathrm{EC}_{50}=50.93 \mu \mathrm{g} / \mathrm{mL}\right)$ and $4-\mathrm{CF}_{3}\left(\mathbf{5 o}, \mathrm{EC}_{50}=108.27 \mu \mathrm{g} /\right.$ $\mathrm{mL}$ ), into the benzene ring group of mono-ether structure compounds $\mathbf{5 j}-\mathbf{5 r}$, culminated in either a significant or non-significant improvement in antibacterial activity; alkyl groups did not evoke a significant improvement over phenyl. Secondly, compared to other substituents, we found that the introduction of fluorine or chlorine (compounds $\mathbf{5 u}$ and $\mathbf{5 v}$ ) at the 4-position of the phenyl group exerted the most significant effect on improving antibacterial activity against Xoo and Xoc; confusingly, continue compounds $\mathbf{5 v}$ to introduce chlorine atom (such as compound $5 \mathrm{~s}$ with 2,4-Cl, $\mathrm{EC}_{50}=116.76 \mu \mathrm{g} / \mathrm{mL}$ ) on the benzene ring second position was overall antibacterial ability has not improved but has declined. Finally, introduction of electron withdrawing group or electron donating group of thioether structure compounds $\mathbf{5 a}-\mathbf{5 i}$ disfavor to their activities.

\section{Conclusions}

In summary, twenty-six novel 1,2,4-oxadiazole derivatives containing 1,3,4-thiadiazol (1,3,4-oxadiazole) and trifluoromethyl pyridine moieties were synthesized based on 1,2,4-oxadiazole pharmacophore. Compound $\mathbf{5 n}$ and 5v exhibited excellent nematocidal activity, superior to leading compound Tioxazafen. What's more notable is that compound $\mathbf{5 v}$ and $\mathbf{5 u}$ showed significantly inhibitory activity against the plant pathogenic bacteria Xoo and Xoc, respectively, higher than commercial bactericide BMT and TDC. The present study demonstrated the

Table 3 Curative and protective activity of compound $5 \mathrm{v}$ against rice bacterial leaf blight

\begin{tabular}{|c|c|c|c|c|}
\hline \multirow[t]{2}{*}{ Treatments } & \multicolumn{2}{|l|}{ Protective activity } & \multicolumn{2}{|l|}{ Curative activity } \\
\hline & Disease index (\%) & Control effect $(\%)^{a}$ & Disease index (\%) & Control effect $(\%)^{\mathrm{a}}$ \\
\hline $5 v$ & 60.6 & $27.6 \pm 3.7$ & 50.1 & $37.8 \pm 4.2$ \\
\hline BMT & 54.2 & $36.1 \pm 2.5$ & 43.3 & $46.7 \pm 1.8$ \\
\hline TDC & 56.5 & $30.9 \pm 3.4$ & 56.2 & $31.8 \pm 5.6$ \\
\hline Negative control & 84.3 & - & 81.2 & - \\
\hline
\end{tabular}

Compounds $\mathbf{5 v}$ at a concentration of $200 \mu \mathrm{g} / \mathrm{mL}$ against rice bacterial leaf blight

a Statistical analysis was conducted using the analysis of variance method 


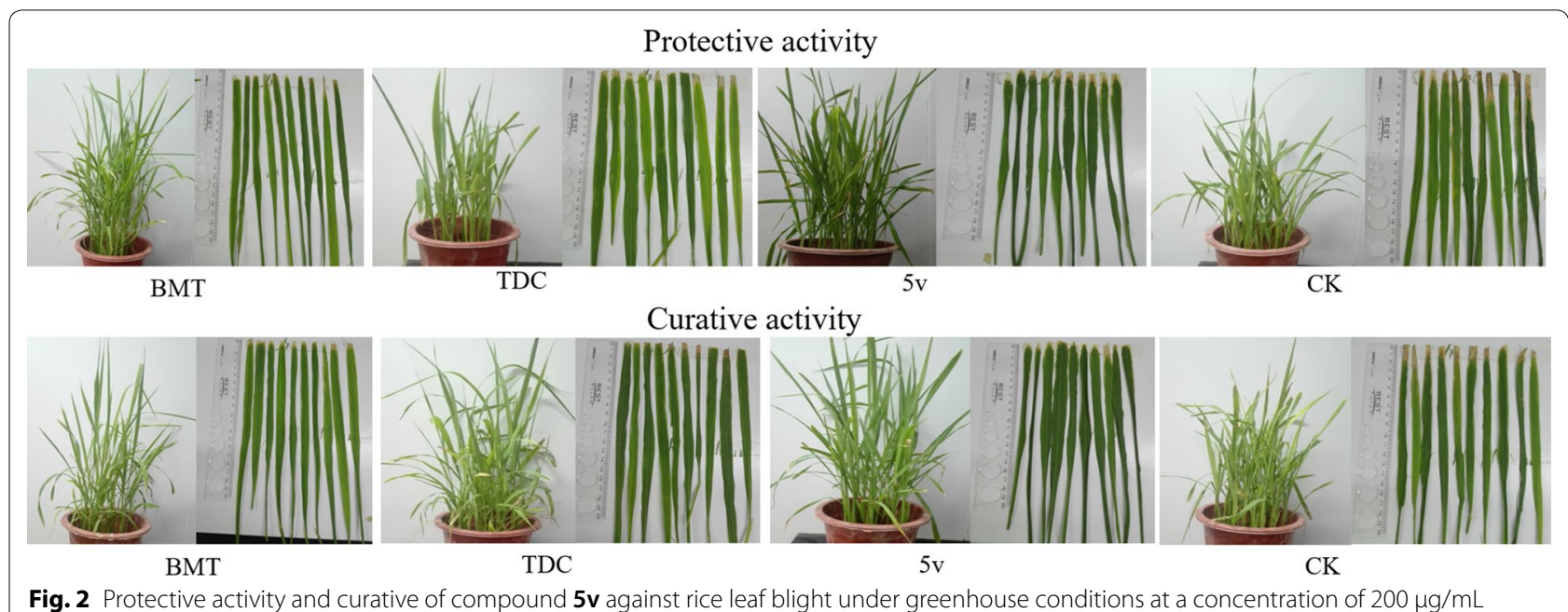

potential of 1,2,4-oxadiazole ether derivatives as effective nematocidal and antimicrobial agents for crop protection and should serve as a basis for future studies.

\section{Supplementary information}

Supplementary information accompanies this paper at https://doi. org/10.1186/s13065-020-00722-1.

Additional file 1. The ${ }^{1} \mathrm{H} N M R,{ }^{13} \mathrm{CNMR}$, and HRMS data of target compounds.

\section{Abbreviations}

Xoo: Xanthomonas oryzae pv. oryzae; Xoc: Xanthomonas oryzae pv. oryzicola; BMT: Bismerthiazol; TDC: Thiodiazole copper; ${ }^{1} \mathrm{H}$ NMR: ${ }^{1} \mathrm{H}$ nuclear magnetic resonance; ${ }^{13} \mathrm{C}$ NMR: ${ }^{13} \mathrm{C}$ nuclear magnetic resonance; HRMS: High resolution mass spectrum.

\section{Acknowledgements \\ Not applicable.}

\section{Authors' contributions}

The current study is an outcome of constructive discussion with BS and XG. $L Z, X G$ and $Q W$ carry out their synthesis and characterization experiments; $L Z$, $H Z, D L, Y F$, and $Q W$ performed the biological activities; $L Z, X G, H Z, D L, Y F$, and QW carried out the ${ }^{1} \mathrm{H} \mathrm{NMR},{ }^{13} \mathrm{C}$ NMR and HRMS spectral analyses; LZ and XG were involved in the drafting of the manuscript and revising the manuscript. All authors read and approved the final manuscript.

\section{Funding}

The authors gratefully acknowledge assistance from the National Key Research Development Program of China (2017YFD0201404 and 2018YFD0200100), the National Nature Science Foundation of China (32060622), the Outstanding Young Scientific and Technological Talents Project of Guizhou Province ([2019]5646), and the Construction Project of Key Laboratories from the Education Department of Guizhou Province (QJHKY [2018] 001).

\section{Availability of data and materials}

All data generated or analysed during this study are included in this published article [and its Additional files].

\section{Ethics approval and consent to participate}

The experimental research was performed following the IUCN Policy Statement on Research Involving Species at Risk of Extinction and the Convention on the Trade in Endangered Species of Wild Fauna and Flora and it was approved by Subcommittee of Experimental Animal Ethics, and Center for Research and Development of Fine Chemicals of Guizhou University.

\section{Consent for publication}

Not applicable.

\section{Competing interests}

The authors declare that they have no competing interests.

Received: 24 April 2020 Accepted: 12 November 2020 Published online: 22 November 2020

\section{References}

1. Zou LF, Li YR, Chen GY (2011) A non-marker mutagenesis strategy to generate poly-hrp gene mutants in the rice pathogen Xanthomonas oryzae pv. oryzicola. Agric Sci China 10:1139-1150

2. Graham JH, Gottwald TR, Cubero J, Achor DS (2004) Xanthomonas axonopodis pv. citri: factors affecting successful eradication of citrus canker. Mol Plant Pathol 5:1-15

3. Yi CF, Chen JX, Wei CQ, Wu SK, Wang SB, Hu DY, Song BA (2020) $a$-Haloacetophenone and analogues as potential antibacterial agents and nematicides. Bioorg Med Chem Lett 30:126814-126818

4. Li P, Hu DY, Xie DD, Chen JX, Jin LH, Song BA (2018) Design, synthesis, and evaluation of new sulfone derivatives containing a 1,3,4-oxadiazole moiety as active antibacterial agents. J Agric Food Chem 66:3093-3100

5. Shi J, Luo N, Ding MH, Bao XP (2019) Synthesis, in vitro antibacterial and antifungal evaluation of novel 1,3,4-oxadiazole thioether derivatives bearing the 6-fluoroquinazolinylpiperidinyl moiety. Chin Chem Lett 31:434-438

6. Abad P, Gouzy J, Aury JM, Castagnone-Sereno P, Danchi EGJ, Deleury E, Perfus-Barbeoch L, Anthouard V, Artiquenave F, Blok VC, Caillaud MC, Coutinho PM, Dasilva C, De Luca F, Deau F, Esquibet M, Flutre T, Goldstone JV, Hamamouch N, Hewezi T, Jaillon O, Jubin C, Leonetti P, Magliano M, Maier TR, Markov GV, McVeigh P, Pesole G, Poulain J, Robinson-Rechavi M, Sallet E, Segurens B, Steinbach D, Tytgat T, Ugarte E, van Ghelder C, 
Veronico P, Baum TJ, Blaxter M, Bleve-Zacheo T, Davis EL, Ewbank JJ, Favery B, Grenier E, Henrissat B, Jones JT, Laudet V, Maule AG, Quesneville H, Rosso MN, Schiex T, Smant G, Weissenbach J, Wincker P (2008) Genomese quence of the metazoan plant-parasitic nematode Meloidogyne incognita. Nat Biotechnol 26:909-915

7. Abad P, Favery B, Rosso MN, Castagnone SP (2003) Root-knot nematode parasitism and host response: molecular basis of a sophisticated interaction. Mol Plant Pathol 4:217-224

8. Djian-Caporalino C, Fazari A, Arguel MJ, Vernie T, VandeCasteele C, Faure I, Brunoud G, Pijarowski L, Palloix P, Lefebvre V, Abad P (2007) Root-knot nematode (Meloidogyne spp.) Me resistance genes in pepper (Capsicum annuum L.) are clustered on the P9 chromosome. Theor Appl Genet 114:473-486

9. Seenivasan N (2017) Status of root-knot nematode, Meloidogyne hapla infection on carrot at Kodaikanal hills of Tamil Nadu, India and its yield loss estimation. Int J Curr Microbiol App Sci 6:1-7

10. Meher HC, Gajbhiye VT, Chawla G, Singh GD (2009) Virulence development and genetic polymorphism in Meloidogyne incognita (Kofoid \& White) Chitwood after prolonged exposure to sublethal concentrations of nematicides and continuous growing of resistant tomato cultivars. Pest Manag Sci 65:1201-1207

11. Caboni P, Aissani N, Cabras T, Falqui AB, Marotta RB, Liori B, Ntalli NK, Sarais G, Sasanelli N, Tocco G (2013) Potent nematicidal activity of phthalaldehyde, salicylaldehyde, and cinnamic aldehyde against Meloidogyne incognita. J Agric Food Chem 61:1794-1803

12. Jang JY, Dang QL, Choi YH, Choi GJ, Jang KS, Cha B, Luu NH, Kim JC (2015) Nematicidal activities of 4-quinolone alkaloids isolated from the aerial part of Triumfetta grandidens against Meloidogyne incognita. J Agric Food Chem 63:68-74

13. Shafikova TN, Omelichkina YV (2015) Molecular-genetic aspects of plant immunity to phytopathogenic bacteria and fungi. Russ J Plant Physiol 62:571-585

14. Qiao M, Ying GG, Singer AC, Zhu YG (2018) Review of antibiotic resistance in China and its environment. Environ Int 110:160-172

15. Pan XY, Xu S, Wu J, Luo JY, Duan YB, Wang JX, Zhang F, Zhou MG (2018) Screening and characterization, of Xanthomonasoryzae pv. oryzae strains with resistance to pheazine-1-carboxylic acid. Pestic Biochem Phys 145:8-14

16. Wang PY, Zhou L, Zhou J, Wu ZB, Xue W, Song BA, Yang S (2016) Synthesis and antibacterial activity of pyridinium-tailored 2,5-substituted-1,3,4oxadiazole thioether/sulfoxide/sulfone derivatives. Bioorg Med Chem Lett 26:1214-1217

17. Xu WM, Li SZ, He M, Yang S, Li XY, Li P (2013) Synthesis and bioactivities of novel thioether/sulfone derivatives containing 1,2,3-thiadiazole and 1,3,4-oxadiazole/thiadiazole moiety. Bioorg Med Chem Lett 23:5821-5824

18. Aslam MN, Mukhtar T, Ashfaq M, Hussain MA (2017) Evaluation of chili germplasm for resistance to bacterial wilt caused by Ralstonia solanacearum. Australas Plant Path 46:289-292

19. Devendar P, Yang GF (2017) Sulfur-containing agrochemicals. Top Curr Chem (Z) 375:82

20. Dahl BH, Peters D, Olsen GM, Timmermann DB, Joergensen S (2006) Novel oxadiazole derivatives as nicotinic acetylcholine receptor modulators and their preparation, pharmaceutical compositions, and use in the treatment of various central nervous system and peripheral nervous system diseases WO $2006114400 \mathrm{~A} 1$

21. Dai H, Chen J, Li G, Ge S, Shi Y, Fang Y, Ling Y (2017) Design, synthesis, and bioactivities of novel oxadiazole-substituted pyrazole oximes. Bioorg Med Chem Lett 27:950-953

22. Liu Q, Zhu R, Gao S, Ma SH, Tang HJ, Yang JJ, Diao YM, Wang HL, Zhu HJ (2017) Structure-based bioisosterism design, synthesis, insecticidal activity and structure-activity relationship (SAR) of anthranilic diamide analogues containing 1,2,4-oxadiazole rings. Pest Manag Sci 73:917-924

23. Sheng R, Li S, Lin GY, Shangguan SH, Gu YC, Qiu N, Cao J, He QJ, Yang B, Hu YZ (2015) Novel potent HIF-1 inhibitors for the prevention of tumor metastasis: discovery and optimization of 3-aryl-5-indazole-1,2,4-oxadiazole derivatives. Rsc Adv 5:81817-81830

24. Takahashi H, Riether D, Bartolozzi A, Bosanac T, Berger V, Binetti R, Broadwater J, Chen ZD, Crux R, Lombaert SD, Dave R, Dines JA, FadraKhan T, Flegg A, Garrigou M, Hao MH, Huber J, Hutzler JM, Kerr S, Kotey A, Liu WM, Lo HY, Loke PL, Mahaney PE, Morwick TM, Napier S, Olague A, Pack E, Padyana AK, Thomson DS, Tye H, Wu LF, Zindell RM, Abeywardane A, Simpson T (2015) Synthesis, SAR, and series evolution of novel oxadiazole-containing 5 -lipoxygenase activating protein inhibitors: discovery of 2-[4-(3-\{(R)-1-[4-(2-amino-pyrimidin-5-yl)-phenyl]1-cyclopropyl-ethyl\}-[1,2,4]oxadiazol-5-yl)-pyrazol-1-yl]-N, N-dimethylacetamide(Bl665915). J Med Chem 58:1669-1690

25. Li P, Shi L, Yang X, Yang L, Chen XW, Wu F, Shi QC, Xu WM, He M, Hu DY, Song BA (2014) Design, synthesis, and antibacterial activity against rice bacterial leaf blight and leaf streak of 2,5-substituted-1,3,4-oxadiazole/ thiadiazole sulfone derivative. Bioorg Med Chem Lett 24:1677-1680

26. Xu WM, Han FF, He M, Hu DY, He J, Yang S, Song BA (2012) Inhibition of tobacco bacterial wilt with sulfone derivatives containing an 1,3,4-oxadiaole. J Agric Food Chem 60:1036-1041

27. Xu WM, He J, He M, Han FF, Chen XH, Pan ZX, Wang J, Tong MG (2011) Synthesis and antifungal activity of novel sulfone derivatives containing 1,3,4-oxadiazole moieties. Molecules 16:9129-9141

28. Chen Q, Zhu XL, Jiang LL, Liu ZM, Yang GF (2008) Synthesis, antifungal activity and CoMFA analysis of novel 1,2,4-triazolo[1,5-a]pyrimidine derivatives. Eur J Med Chem 43:595-603

29. Zhang MZ, Mulholland N, Beattie D, Irwin D, Gu YC, Chen Q, Yang GF, Clough $J$ (2013) Synthesis and antifungal activity of 3-(1,3,4-oxadiazol5-yl)-indoles and 3-(1,3,4-oxadiazol-5-yl)methyl-indoles. Eur J Med Chem 63:22-32

30. Chen JX, Chen YZ, Gan XH, Song BJ, Hu DY, Song BA (2018) Synthesis, nematicidal evaluation, and 3D-QSAR analysis of novel 1,3,4-oxadiazolecinnamic acid hybrids. J Agric Food Chem 66:9616-9623

31. Abad-Feuntes A, Ceballos-Alcantrallia E, Mercader JV, Agulló C, AbadSomovilla A, Esteve-Turrillas FA (2015) Determination of succinate dehydrogenase-inhibitor fungicide residues in fruits and vegetables by liquid-chromatography-tandem mass spectrometry. Anal Bioanal Chem 407:4207-4211

32. Jones JG, Kleczewski NM, Desaeger J, Meyer SL, Johnson GC (2017) Evaluation of nematicides for southern root-knot nematode management in lima bean. Crop Protect 96:151-157

33. Chen JX, Yi CF, Wang SB, Wu SK, Li SY, Hu DY, Song BA (2019) Novel amide derivatives containing 1,3,4-thiadiazole moiety: design, synthesis, nematocidal and antibacterial activities. Bioorg Med Chem Lett 29:1203-1210

34. Fan ZJ, Shi J, Luo N, Ding MH, Bao XP (2019) Synthesis, crystal structure, and agricultural antimicrobial evaluation of novel quinazoline thioether derivatives incorporating the 1,2,4-triazolo[4,3-a]pyridine moiety. J Agric Food Chem 67:11598-11606

35. Jung J, Zhao YY (2013) Impact of the structural differences between $a$ - and $\beta$-chitosan on their depolymerizing reaction and antibacterial activity. J Agric Food Chem 61:8783-8789

36. Zhu XF, Xu Y, Peng D, Zhang Y, Huang TT, Wang JX, Zhou MG (2013) Detection and characterization of bismerthiazol-resistance of Xanthomonas oryzae pv. oryzae. Crop Prot 47:24-29

37. Wang MW, Zhu HH, Wang PY, Zeng D, Wu YY, Liu LW, Wu ZB, Li Z, Yang $S$ (2019) Synthesis of thiazolium-labeled 1,3,4-oxadiazole thioethers as prospective antimicrobials: in vitro and in vivo bioactivity and mechanism of action. J Agric Food Chem 67:12696-12708

\section{Publisher's Note}

Springer Nature remains neutral with regard to jurisdictional claims in published maps and institutional affiliations. 